\title{
Identification of Novel LncRNA Biomarkers and Construction of LncRNA-Related Networks in Han Chinese Patients with Ischemic Stroke
}

\author{
Xiaojing Guo ${ }^{a}$ Jialei Yang ${ }^{a}$ Baoyun Liang ${ }^{b}$ Tingting Shen ${ }^{b}$ Yan Yan ${ }^{b}$ \\ Siyun Huang ${ }^{b}$ Jinying Zhou ${ }^{b}$ Jiao Huang ${ }^{b}$ Lian Gu ${ }^{b} \quad$ Li Su $^{a}$ \\ aSchool of Public Health, Guangxi Medical University, Nanning, bFirst Affiliated Hospital of Guangxi \\ University of Chinese Medicine, Nanning, China
}

\section{Key Words}

Ischemic stroke $\bullet$ LncRNA $・$ Microarray $•$ Biomarker $•$ Network analysis

\begin{abstract}
Background/Aims: Long non-coding RNAs (IncRNAs) are potential biomarkers of tumors, cardiac disease, and cerebral disease because of their interaction with coding RNAs. This work focused on ischemic stroke (IS) and aimed to identify novel IncRNA biomarkers and construct IncRNA-related networks in IS. Methods: Differentially expressed IncRNAs were identified using Arraystar Human LncRNA Microarray v4.0, and validated with qRT-PCR. A IncRNA-mRNA co-expression network and a IncRNA-miRNA-mRNA regulatory network were constructed. Functional and pathway analyses were then performed. Results: In total, 560 up-regulated and 690 down-regulated differentially expressed IncRNAs were found $(P<$ 0.05 , false discovery rate $<0.05$, absolute fold change $\geq 2$ ). qRT-PCR results confirmed that InCRNA-ENST00000568297, IncRNA-ENST00000568243, and IncRNA-NR_046084 exhibited significant differential expression between IS and controls (all $P<0.05$ ). Areas under the curves (AUCs) for these IncRNAs were $0.733,0.743$, and 0.690 , respectively, and the combined AUC was 0.843. A coding-noncoding co-expression (CNC) network was constructed based on Pearson's correlation coefficient. A specific IncRNA-miRNA-mRNA regulatory network of ENST00000568297, ENST00000568243, and NR_046084 was also constructed. Functional annotation of the up- and down-regulated mRNAs was performed. Pathway analysis enriched IS-related pathways with mRNAs in the IncRNA-miRNA-mRNA regulatory network. Conclusion: LncRNA and mRNA expression profiles in human peripheral blood were altered after IS. ENST00000568297, ENST00000568243, and NR_046084 were identified as novel potential diagnostic biomarkers of IS. Analysis of the CNC network and IncRNA-miRNAmRNA regulatory network suggested that IncRNAs may participate in IS pathophysiology by regulating pivotal miRNAs, mRNAs, or IS-related pathways.

X. Guo, J. Yang, B. Liang and T. Shen contributed equally to this work. Published by S. Karger AG, Basel \begin{tabular}{ll}
\hline Lian Gu & Department of Internal Neurology, First Affiliated Hospital of Guangxi University of Chinese Medicine, Nanning, 530023; \\
and Li Su & Department of Epidemiology, School of Public Health, Guangxi Medical University, Nanning, 530021 (China) \\
& Tel. +86 07715848507, Fax +86 07715848507, E-Mail gulian2012@163.com; suli2018@hotmail.com
\end{tabular}
\end{abstract}




\section{Cellular Physiology Cell Physiol Biochem 2018;50:2157-2175

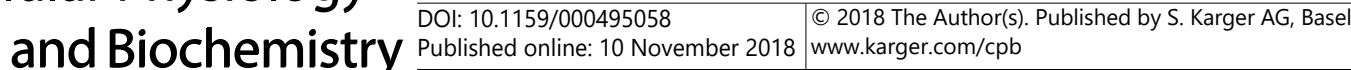 \\ Guo et al.: LncRNA Biomarkers and Networks in Chinese IS Patients}

\section{Introduction}

Ischemic stroke (IS) is a neurological impairment generated by focal cerebral, spinal, or retinal infarction. IS constitutes about $85 \%$ of stroke and is the second leading of cause death and disability worldwide [1]. As reported by the Global Burden of Disease Study 2013, death due to IS in developing countries amounted to $75 \%$ of the total morbidity rate worldwide; meanwhile, the disability adjusted of life years (DALY) in developing countries amounted to $81 \%$ of the total DALY worldwide [2]. In the years to come, the prevalence of IS and the number of patients are predicted to decrease in high-income countries and double in lowand middle-income countries [3]. China, as a middle-income developing country, bears a heavy burden of stroke. Therefore, research on IS among Chinese is continuing and necessary.

Long non-coding RNAs (lncRNAs) have a long transcription sequence between 200 nucleotides and $100 \mathrm{~kb}$, with minimal coding power and incomplete annotation [4]. Great progress in the field of IncRNAs has been achieved with the development of novel methodologies using a composite algorithm and databases [5]. In the past few years, lncRNAs have emerged as key regulators of various biological or cellular processes [6]. LncRNA profiles have been identified through microarray and high-throughput sequencing analyses to determine the regulatory function of lncRNAs in diseases. Dharap performed microarray analysis of rat cerebral cortex and revealed that lncRNA expression profiles are significantly altered after stroke [7]. Wu [8] and Dharap [9] performed microarray analysis of rat brain tissues to identify and explore the potential function of IncRNA-N1LR. Bhattarai performed high-throughput sequencing in rat cerebral cortex and discovered novel stroke-associated IncRNAs [10]. Zhang performed RNA sequencing and confirmed that lncRNA transcriptomic profiles in mouse brain microvascular endothelial cells changed after cerebral ischemia [11]. High-throughput analyses (microarray and sequencing) in vitro (oxygen glucose deprivation model in cell) or in vivo (middle cerebral artery occlusion model of rat or mouse) indicated that lncRNA probably participates in the pathogenesis of stroke. However, analysis of human samples is essential to explore the association between lncRNAs and stroke in patients with this disease.

Dykstra-Aiello performed microarray analysis and identified 299 and 97 differentially expressed IncRNAs in the peripheral blood of male and female patients with stroke compared with controls $(P<0.005$, absolute fold change $(|\mathrm{FC}|)>1.2)[12]$. However, qRT-PCR analysis was not performed to verify the expression of the identified differentially expressed IncRNAs. Therefore, distinctly expressed lncRNAs between patients with IS and controls must be identified in human peripheral blood for subsequent determination of IncRNA biomarkers and exploration of functional mechanism.

In this study, microarray analysis was performed to identify the expression profiles of lncRNAs and mRNAs in human peripheral blood of patients with IS and control subjects. Nine differentially up-regulated IncRNAs from the microarray were validated through qRT-PCR analysis. The sample size was expanded for further validation and identification of IncRNA biomarkers for IS diagnosis. A coding-noncoding (CNC) co-expression network and a lncRNA-miRNA-mRNA regulatory network were constructed through bioinformatics analysis.

\section{Materials and Methods}

\section{Study subjects and blood collection}

Patients with IS were recruited from the Encephalopathy Department of the First Affiliated Hospital of Guangxi University of Chinese Medicine in Nanning from January 2016 to June 2017. The patients were diagnosed by head magnetic resonance imaging (MRI) and computed tomography (CT) scanning with IS diagnostic criteria according to the Fourth Chinese National Conference on cerebrovascular diseases. The patients had no hemorrhagic stroke, transient ischemic attack, cancer, trauma, arterial inflammation, cerebral 


\section{Cellular Physiology Cell Physiol Biochem 2018;50:2157-2175

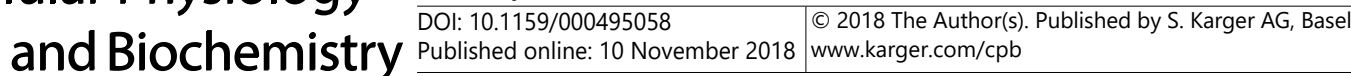

vascular malformation, or aneurysm. Healthy controls were also recruited from two health service centers, namely, Health Service Center of Jinzhou Community and Health Service Center of Dongge Community in Nanning. Healthy controls were matched with patients with IS in terms of age and sex composition and had no history of cerebrovascular or autoimmune diseases. All participants were of Chinese Han descent and were not related to each other. This study was conducted according to the Declaration of Helsinki and approved by the Institutional Review Board of Guangxi University of Chinese Medicine. Written informed consent was obtained from all participants prior to the study.

Whole blood samples were obtained though venipuncture using PAXgene Blood RNA tubes (PreAnalytiX, GmbH, Switzerland) to stabilize blood RNA. Samples for lncRNA microarray analysis were obtained from 10 patients with IS and 10 healthy controls. The samples were subjected to the first-stage validation of IncRNA expression level. IS and control subjects $(n>50)$ were used for validation and receiver operating characteristic (ROC) curve analysis. Peripheral blood was collected after symptom onset within $72 \mathrm{~h}$. Fasting blood from healthy controls was also collected strictly. All collected blood samples were kept cold during transport to laboratory within $2 \mathrm{~h}$ and stored at $-80^{\circ} \mathrm{C}$.

\section{RNA extraction, labeling and hybridization}

RNA was isolated with PAXgene blood RNA isolation kits (Qiagen, Hilden, Germany) according to the manufacturer's protocol by using TRI Reagent BD (Molecular Research Center, Inc., Cincinnati, OH) for microarray and TRIzol reagents (Invitrogen, Carlsbad, CA) for qRT-PCR analysis. RNA quantity and quality were measured using NanoDrop ND-1000 and TECAN Spark 10M, respectively. Standard denaturing agarose gel electrophoresis was performed to determine RNA integrity. Isolated RNAs were stored at $-80^{\circ} \mathrm{C}$ prior to experiments. Sample labeling and array hybridization were performed according to the Agilent One-Color Microarray-Based Gene Expression Analysis protocol (Agilent Technologies, Santa Clara, CA) with minor modifications. In brief, mRNA was purified from total RNA after removal of rRNA (mRNA-ONLY Eukaryotic mRNA Isolation Kit, Epicentre Madison, WI). Each sample was amplified and transcribed into fluorescent cRNA along the entire length of the transcripts without $3^{\prime}$ bias by utilizing a random priming method (Arraystar Flash RNA Labeling Kit, Arraystar Rockville, MD). The labeled cRNAs were purified by RNeasy Mini Kit (Qiagen). Hybridization solution $(50 \mu \mathrm{L})$ was dispensed into the gasket slide and assembled to the IncRNA expression microarray slide. The slides were incubated in an Agilent hybridization oven at $65^{\circ} \mathrm{C}$ for $17 \mathrm{~h}$. The hybridized arrays were washed, fixed, and scanned with the Agilent DNA Microarray Scanner (part number G2505C).

\section{LncRNA and mRNA microarray}

Analysis was performed using Arraystar Human LncRNA Microarray v4.0 provided by KangChen Bio-Tech, Inc (Shanghai, China). A total of 40, 173 IncRNAs and 20, 730 coding transcripts were detected. The acquired array images were evaluated using Agilent Feature Extraction software (version 11.0.1.1). Quantile normalization and subsequent data processing were performed using the GeneSpring GX v12.1 software package (Agilent Technologies). LncRNAs and mRNAs that showed statistically significant differential expression between IS and control groups were identified through $P$ value, false discovery rate FDR, and FC filtering. Hierarchical clustering was performed using GeneSpring GX v12.1.

\section{qRT-PCR}

In brief, cDNAs were synthesized from total RNA by using SuperScript III Reverse Transcriptase (Invitrogen) for stage one qRT-PCR or the PrimerScript RT reagent kit with gDNA Eraser (Takara, Beijing, China) for stages two and three qRT-PCR. Primers for IncRNAs were

Table 1. PCR primers of the 9 significantly upregulated IncRNAs from the microarray

\begin{tabular}{lc}
\hline Gene name & Primers \\
\hline 3-actin (H) & F:5' GTGGCCGAGGACTTTGATTG 3' \\
& R:5' CCTGTAACAACGCATCTCATATT 3' \\
T008148 & F:5' ATGAGCGAAAGGGGTTGGT 3' \\
& R:5' TCTGGCTGAAGGGTAGAGTGAC 3' \\
TCONS_00008492 & F:5' GCACCAGAGGGATCAACTCA 3' \\
& R:5' TCCTTTGCAACCAGGTCTACTT 3' \\
ENST00000568297 & F:5' GGGAGCTGTCCTATAAATCTTG 3' \\
& R:5' AAAGCAAGAAGCAAAACTACATC 3' \\
ENST00000442403 & F:5' TATTGAACCCTGCCTCCCA 3' \\
& R:5' GAATCTCTGTGAGACCAGTCATG 3' \\
ENST00000565098 & F:5' CAGAGGAAACAGATGACTTGAGG 3' \\
& R:5' GGGTTGTGGGAAGGAACTAAA 3' \\
ENST00000568243 & F:5' GCTGCTCCTTATTTCTCCTGTAG 3' \\
& F:5' CGCTCTGTTAGGTTGCATGG 3' \\
ENST00000467369 & F:5' ACCCTCCTCCTGCTGTTCTC 3' \\
& R:5' ACAATGGCTCACACCTGTAATG 3' \\
NR_046084 & F:5' GCCAAGGAGACTGTTCTGATG 3' \\
& R:5' GGTTGTTTCCAAGTGCTGTTC 3' \\
NR_033861 & F:5' GGAGGTGGCATCCAGATTT 3' \\
\hline
\end{tabular}




\section{Cellular Physiology Cell Physiol Biochem 2018;50:2157-2175 \begin{tabular}{l|l|l} 
and Biochemistry Published online: 10 November 2018 & $\begin{array}{l}\text { (c) } 2018 \text { The Author(s). Published by S. Karger AG, Basel } \\
\text { www.karger.com/cpb }\end{array}$ \\
\hline
\end{tabular}

designed using Primer Premier v5.0 software and checked with the NCBI Basic Local Alignment Search Tool (Table 1).

Stage one qRT-PCR. SYBR Green 2X Real-Time qPCR Master Mix (Arraystar) was used in the ViiA 7 RealTime PCR System (Applied Biosystems). Stage one qRT-PCR was performed as follows: $95^{\circ} \mathrm{C}$ for $10 \mathrm{~min} ; 40$ PCR cycles of $95^{\circ} \mathrm{C}$ for $10 \mathrm{~s}$ and $60^{\circ} \mathrm{C}$ for $60 \mathrm{~s}$ for fluorescence collection); amplification at $95^{\circ} \mathrm{C}$ for $10 \mathrm{~s}, 60^{\circ} \mathrm{C}$ for $60 \mathrm{~s}$, and $95^{\circ} \mathrm{C}$ for $\left.15 \mathrm{~s}\right)$; and slow heating at $99^{\circ} \mathrm{C}\left(0.05^{\circ} \mathrm{C} / \mathrm{s}\right.$ automatically) to obtain the melting curve.

Stage two and stage three qRT-PCR. Sample collection was expanded to 50 patients with IS and 50 controls for stage two validation. At stage three validation, sample collection was expanded to at least 80 patients with IS and 80 controls. SYBR Premix Ex Taq II (Takara, China) was used in the ABI 7500 Fast Real-Time PCR System (Applied Biosystems, USA) or the ABI StepOnePlus Real-Time PCR System (Applied Biosystems). The procedures for stages two and three qRT-PCR were as follows: $95^{\circ} \mathrm{C}$ for $30 \mathrm{~s} ; 40$ PCR cycles of $95^{\circ} \mathrm{C}$ for $5 \mathrm{~s}$ and $60^{\circ} \mathrm{C}$ for $34 \mathrm{~s}$ for fluorescence collection; amplification at $95^{\circ} \mathrm{C}$ for $15 \mathrm{~s}, 60^{\circ} \mathrm{C}$ for $60 \mathrm{~s}, 95^{\circ} \mathrm{C}$ for $15 \mathrm{~s}$, and $60^{\circ} \mathrm{C}$ for $15 \mathrm{~s}$ to obtain the melting curve.

The threshold cycle value $(\mathrm{Ct})$ of each product was determined. Expression levels were calculated using the $2^{-\Delta c t}$ method and normalized to the internal control of $\beta$-actin. LncRNA levels in patients with IS were expressed as FC against the average level of the same lncRNA in controls.

\section{Bioinformatic analysis}

GO functional annotation and KEGG pathway analysis. Gene ontology (GO) functional annotation and Kyoto Encyclopedia of Genes and Genomes (KEGG) pathway analysis were performed using DAVID Bioinformatics Resources v6.8 online tools (http://david.abcc.ncifcrf.gov/).

Construction of co-expression network. A CNC network was constructed according to Pearson's correlation coefficient (PCC), which represents the correlation of differentially expressed lncRNAs and mRNAs. PCC was calculated for each pair of genes, and significantly correlated pairs with PCC $\geq 0.90$ and $P<0.05$ were selected for construction of the CNC network. The open-source bioinformatics software Cytoscape (version 3.4.0) was used to draw the network. Degree centrality (the number of links between nodes) of IncRNA/mRNA within the CNC network determined the relative importance of the nodes.

Construction of the predicted IncRNA-miRNA-mRNA regulatory network. A comprehensive search in five databases (DIANA-LncBase v2.0, IncRNASNP v2, IncREG, RegRNA v2.0, and LNCipedia v4.1) was performed to predict target miRNAs of IncRNAs. We searched the DIANA-Tarbase v7.0 database (http:// www.microrna.gr/tarbase) to obtain the targeted mRNAs of the predicted miRNAs.

\section{Statistical analysis}

Data analysis and plotting were performed with IBM SPSS Statistics 18.0 software (SPSS Inc., Chicago, IL, USA) and GraphPad Prism 5.0 (GraphPad Software, La Jolla, CA), respectively. Comparison among groups of quantitative variables was performed using the independent-samples $t$ test (normal distribution) or the Mann-Whitney $U$ test (abnormal distribution). Comparison among groups of enumeration data was performed using the $\chi^{2}$ test. Correlation analysis between lncRNA expression and clinical parameters was performed using Pearson's correlation. The area under the receiver operating characteristic (ROC) curves and the calculated area under the ROC curves (AUC) were estimated using SPSS Statistics 18.0 with sensitivity (Se) and specificity (Sp) at the cut-off point to determine the feasibility of diagnosing IS in peripheral whole blood. Significance was assigned at the $P<0.05$ level.

\section{Results}

\section{Clinical characteristics}

Demographic data are shown in Table 2. Healthy controls were comparable with patients with IS. However, the patients and the controls significantly differed in terms of systolic blood pressure (SBP) $(P=0.002)$. 


\section{Cellular Physiology Cell Physiol Biochem 2018;50:2157-2175 and Biochemistry Published online: 10 November $2018 \begin{aligned} & \text { ㄷ } 2018 \text { The Author(s). Published by S. Karger AG, Basel } \\ & \text { www.karger.com/cpb }\end{aligned}$

\section{LncRNA and mRNA Expression profiles of IS}

LncRNA expression profile of IS. A total of 1, 250 differentially expressed lncRNAs obtained from peripheral blood were compared between patients with IS $(n=10)$ and healthy controls $(n=10)$ by using the criteria of absolute fold change $(|\mathrm{FC}|) \geq 2, P$ value $<0.05$, and false discovery rate (FDR) $<0.05$. Among the differentially expressed lncRNAs between the IS patients and healthy participants, 560 were up-regulated and 690 were downregulated. The top 25 (sorted by $|\mathrm{FC}|, P$, and FDR) differentially expressed lncRNAs in overall samples are shown in Table 3. Another top 25 (sorted by $|\mathrm{FC}|, P$, and FDR) differentially expressed lncRNAs in male/female samples are shown in Table S1-S2 (For all supplemental material see www.karger.com/10.1159/000495058/). Hierarchical clustering analysis was performed in 20 samples (10 patients with IS and 10 healthy controls). The results showed distinguishable lncRNA expression profiles (Fig. 1A). Fig. 1B and C present the scatter and volcano plots which were visualized to assess IncRNA expression variation between the two sample groups.

A forward analysis of distinguished IncRNAs based on their categorization was performed. All lncRNAs were classified into seven groups. Except for the undefined IncRNAs $(66.21 \%)$, the highest proportion of IncRNAs belong to intergenic groups (21.81\%), followed by natural antisense (4.95\%), intronic antisense $(4.07 \%)$, intron senseoverlapping (1.44\%), bidirectional (1.12\%), and exon senseoverlapping $\quad(0.40 \%)$ groups (Fig. 2A). The pie

Table 2. Clinical characteristics of participants. Data are mean value \pm standard deviation. IS, ischemic stroke; TC, total cholesterol; TG, triglycerides; HDL, high density lipoprotein; LDL, low density lipoprotein. * To test the statistical significance of the comparisons between groups (healthy controls vs. IS patients), the independent-samples $t$ test (normal distribution) or the Mann-Whitney U test (abnormal distribution) were used for quantitative variables and the $\chi^{2}$ test was used for enumeration data

\begin{tabular}{lccc}
\hline \multirow{2}{*}{ Terms } & \multicolumn{3}{c}{ Samples for microarray and stage one validation } \\
& Healthy control $(\mathrm{n}=10)$ & IS patients $(\mathrm{n}=10)$ & $\mathrm{P}^{*}$ \\
\hline Age \pm years & $69.3 \pm 5.9$ & $63.2 \pm 10.7$ & 0.132 \\
Sex (male/female) & $7 / 3$ & $7 / 3$ & 1.000 \\
Systolic blood pressure (mmHg) & $128.0 \pm 12.1$ & $156.2 \pm 21.9$ & 0.002 \\
Diastolic blood pressure (mmHg) & $78.4 \pm 8.8$ & $88.2 \pm 12.6$ & 0.059 \\
TC (mmol/L) & $5.0 \pm 0.9$ & $5.2 \pm 1.3$ & 0.766 \\
TG (mmol/L) & $1.6 \pm 0.8$ & $1.9 \pm 1.1$ & 0.510 \\
HDL (mmol/L) & $1.2 \pm 0.2$ & $1.1 \pm 0.3$ & 0.766 \\
LDL (mmol/L) & $3.1 \pm 0.8$ & $3.4 \pm 0.8$ & 0.420 \\
Glucose (mmol/L) & $4.6 \pm 0.6$ & $6.2 \pm 3.0$ & 0.073 \\
\hline
\end{tabular}

Table 3. Top 25 of differently expressed lncRNAs in IS patients compared to healthy controls (sorted by $|\mathrm{FC}|)$. $|\mathrm{FC}|$, absolute fold change; FDR, false discovery rate

\begin{tabular}{|c|c|c|c|c|c|c|c|c|c|}
\hline \multicolumn{5}{|l|}{ Up-regulated lncRNAs } & \multicolumn{5}{|c|}{ Down-regulated lncRNAs } \\
\hline Seqname & Gene symbol & $|\mathrm{FC}|$ & $P$ & FDR & Seqname & Gene symbol & $|\mathrm{FC}|$ & $P$ & FDR \\
\hline T008148 & G001747 & 8.187 & 0.001 & 0.010 & NR_027076 & LINC00928 & 17.393 & $<0.001$ & 0.001 \\
\hline TCONS_00008492 & XLOC_003959 & 8.040 & 0.003 & 0.022 & NR_033851 & SERPINB9P1 & 7.227 & $<0.001$ & 0.005 \\
\hline ENST00000568297 & RP11-386M24.6 & 7.868 & 0.011 & 0.047 & TCONS_00015989 & XLOC_007368 & 7.079 & 0.002 & 0.018 \\
\hline ENST00000442403 & AC007064.24 & 7.726 & 0.001 & 0.012 & TCONS_00029193 & XLOC_013852 & 6.350 & $<0.001$ & 0.002 \\
\hline T285334 & G066582 & 6.738 & 0.011 & 0.047 & ENST00000596781 & СТВ-50E14.4 & 6.327 & $<0.001$ & 0.001 \\
\hline ENST00000565098 & RP11-52401.4 & 6.432 & $<0.001$ & 0.004 & T250117 & G057505 & 6.221 & $<0.001$ & 0.001 \\
\hline Т361606 & G085412 & 6.221 & 0.005 & 0.028 & NR_105049 & C5orf66-AS1 & 5.974 & $<0.001$ & 0.002 \\
\hline ENST00000424837 & AP000282.3 & 6.092 & 0.002 & 0.015 & T3̈77137 & G089185 & 5.714 & $<0.001$ & 0.001 \\
\hline T234995 & G054048 & 5.824 & 0.005 & 0.028 & NR_110052 & LOC101929106 & 5.707 & 0.001 & 0.012 \\
\hline ENST00000568243 & RP11-408H20.1 & 5.641 & 0.007 & 0.034 & T246988 & G056794 & 5.556 & $<0.001$ & 0.004 \\
\hline T024358 & G005305 & 5.460 & $<0.001$ & 0.004 & NR_121674 & ERICH6-AS1 & 5.509 & 0.003 & 0.021 \\
\hline Т348942 & G082295 & 5.447 & 0.008 & 0.037 & Т0̄81704 & G018886 & 5.489 & $<0.001$ & 0.002 \\
\hline T162386 & G037535 & 5.397 & $<0.001$ & 0.003 & T221263 & G051177 & 5.462 & $<0.001$ & 0.012 \\
\hline TCONS_00000918 & XLOC_000160 & 5.350 & 0.007 & 0.036 & NR_034023 & LOC339807 & 5.454 & $<0.001$ & 0.037 \\
\hline ENST00000404689 & RP11-127B16.1 & 5.322 & $<0.001$ & 0.006 & TCONS_00015176 & XLOC_007223 & 5.355 & $<0.001$ & 0.014 \\
\hline T076452 & G017605 & 5.053 & $<0.001$ & 0.004 & NR_117097 & LINC01353 & 5.311 & 0.002 & 0.016 \\
\hline T268717 & G062219 & 5.002 & $<0.001$ & 0.007 & ENST00000598616 & AC007193.8 & 5.305 & $<0.001$ & 0.002 \\
\hline T172822 & G040151 & 4.996 & 0.011 & 0.046 & T290706 & G067949 & 5.055 & $<0.001$ & 0.006 \\
\hline uc. $402+$ & uc.402 & 4.807 & 0.002 & 0.016 & uc.363- & uc.363 & 4.951 & 0.012 & 0.049 \\
\hline T202060 & G046681 & 4.802 & 0.005 & 0.027 & NR_033999 & LOC100129148 & 4.930 & $<0.001$ & 0.002 \\
\hline T184924 & G042451 & 4.614 & $<0.001$ & 0.003 & ENST00000456932 & DNM1P51 & 4.882 & $<0.001$ & 0.002 \\
\hline T273869 & G063597 & 4.521 & $<0.001$ & 0.005 & T276638 & G064277 & 4.875 & $<0.001$ & $<0.001$ \\
\hline T055645 & G012808 & 4.489 & $<0.001$ & 0.004 & Т001293 & G000157 & 4.773 & $<0.001$ & 0.001 \\
\hline NR_120309 & LOC102724467 & 4.478 & $<0.001$ & 0.003 & Т299627 & G070053 & 4.752 & $<0.001$ & $<0.001$ \\
\hline Т07 4200 & G017046 & 4.378 & 0.003 & 0.021 & NR_121634 & FOXD3-AS1 & 4.586 & $<0.001$ & 0.004 \\
\hline
\end{tabular}




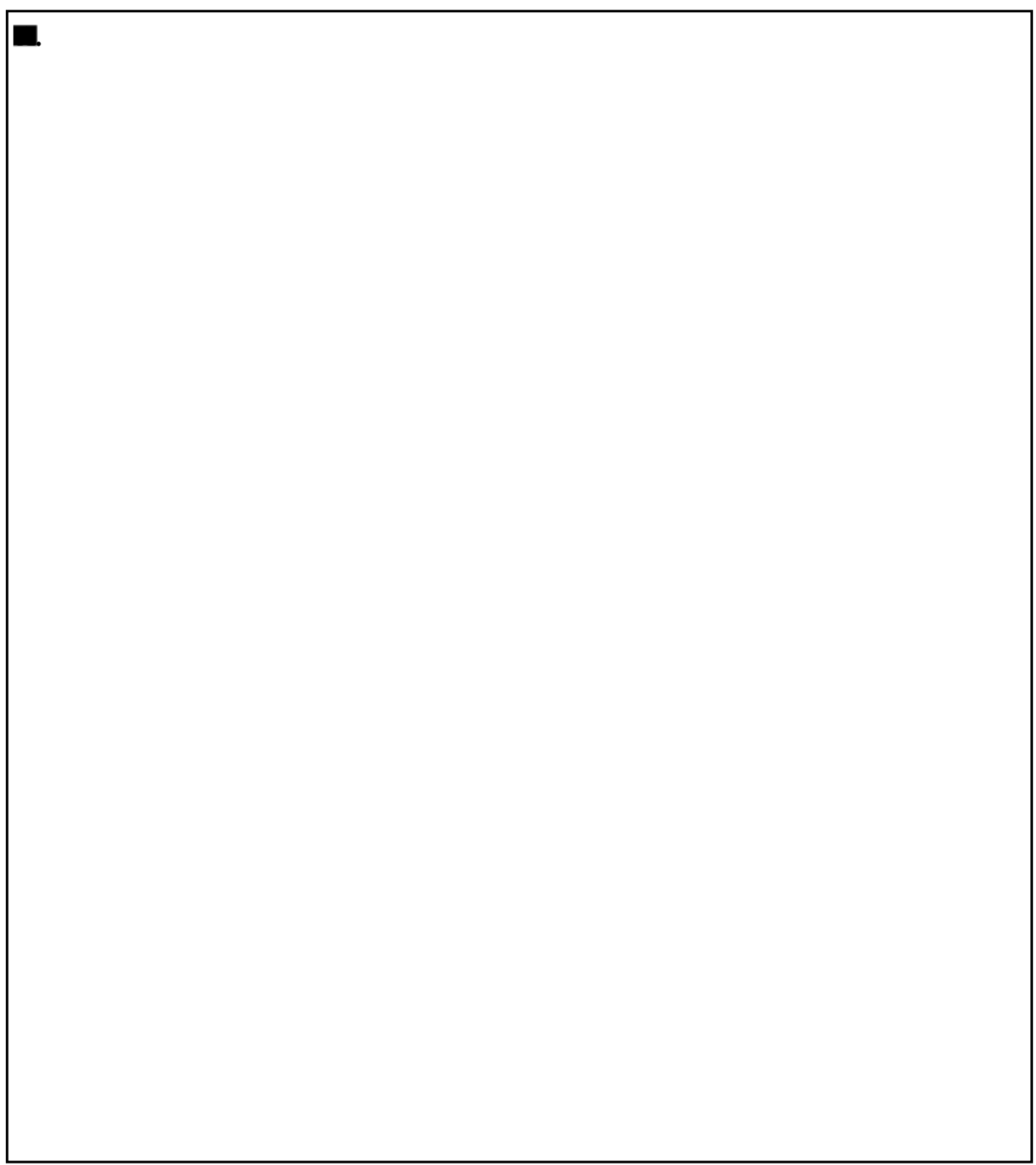

Fig. 1. Differential expression of IncRNAs in IS patients $(n=10)$ and healthy controls $(n=10)$. (A) Heatmap of 1,250 differentially expressed lncRNAs between the two groups. (B) Scatter plot of differential lncRNA expression. X-axis: Test, Y-axis: Control. (C) Volcano plot of differential lncRNA expression. X-axis: $\log _{2}$ fold change (FC) (Test vs Control), Y-axis: $-\log _{10}$ (P value) for each probe. Each lncRNA is represented by a dot, up-regulated as red dots $(\mathrm{FC} \geq 2, \mathrm{P}<0.05, \mathrm{FDR}<0.05)$ and down-regulated as green dots $(\mathrm{FC} \leq-2, \mathrm{P}<0.05$, FDR $<0.05)$. The black dots in the center area represent the remaining $\operatorname{lncRNAs}(-2<\mathrm{FC}<2, \mathrm{P}<0.05$, FDR $<0.05)$.

charts show the components of the up-/down-regulated lncRNAs in each category (Fig. 2B, C). The chromosome and length distribution of dysregulated lncRNAs are shown in Fig. 3A and B. LncRNAs concentrated in few chromosomes and possessed length of less than 4, 000 nt. The distribution of the up-regulated and down-regulated lncRNAs was parallel. 
Fig. 2. Classification of IncRNAs into 7 categories (bidirectional, exon sense-overlapping, intergenic, intron sense-overlapping, intronic antisense, natural antisense, and unknown) according to their relationships with proteincoding genes. (A) Numbers of identified lncRNAs in different categories. (B) Pie charts showing the components of up-regulated lncRNAs in each category. (C) Pie charts showing the components of down-regulated IncRNAs in each category.

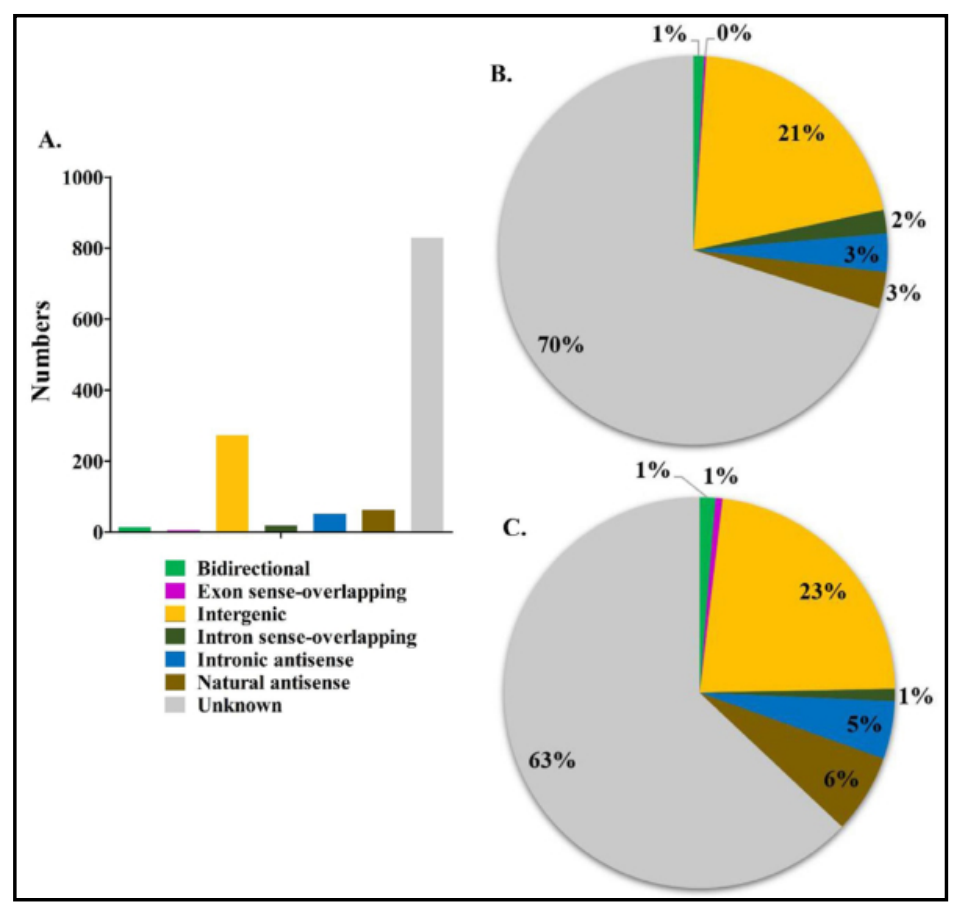

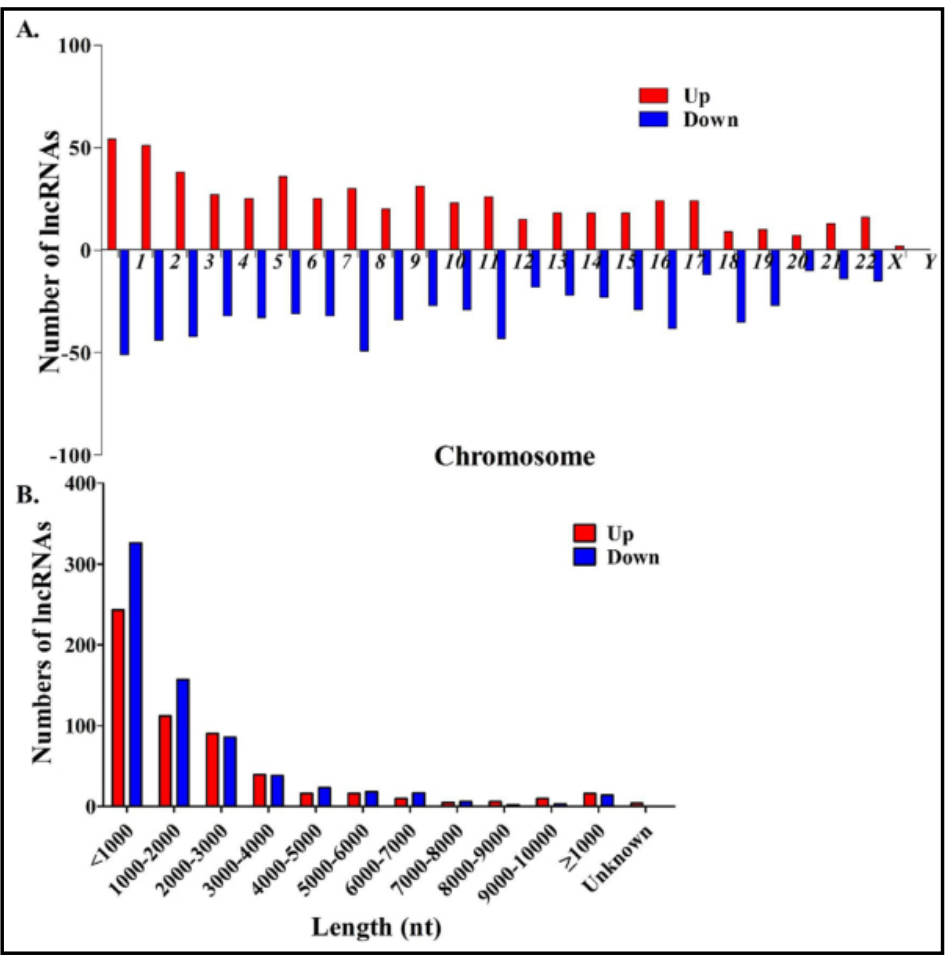

Fig. 3. Chromosome and length distribution of dysregulation lncRNAs. (A) Chromosome distribution of up- and down-regulated lncRNAs. (B) Length distribution of up- and down-regulated IncRNAs.

Expression profile of $m R N A$ in IS. In the same cohort of patients with IS ( $n$ $=10$ ) and healthy controls $(n=10)$, we screened 733 significantly differentially expressed mRNAs through microarray analysis based on the criteria of $|\mathrm{FC}| \geq$ 2.0, $P<0.05$, and FDR $<$ 0.05 (Fig. 4A). Of these 733 mRNAs, 249 were upregulated and 484 were down-regulated. The top 25 differentially expressed mRNAs in all samples are presented in Table 4. In addition, the top 25 (sorted by $|\mathrm{FC}|, P$, and FDR) differentially expressed mRNAs in male and female samples are shown in Table S3 and S4. Fig. 4B and C show scatter and volcano plots, respectively, for assessment of variation in mRNA expression between the patients with IS and the healthy controls. The potential functions of differentially expressed lncRNAs were predicted by functional annotation for dysregulated mRNAs. Significantly up-regulated and down-regulated mRNAs in the microarray (setting $|\mathrm{FC}| \geq 2, P<0.05, \mathrm{FDR}<0.05$ ) were 


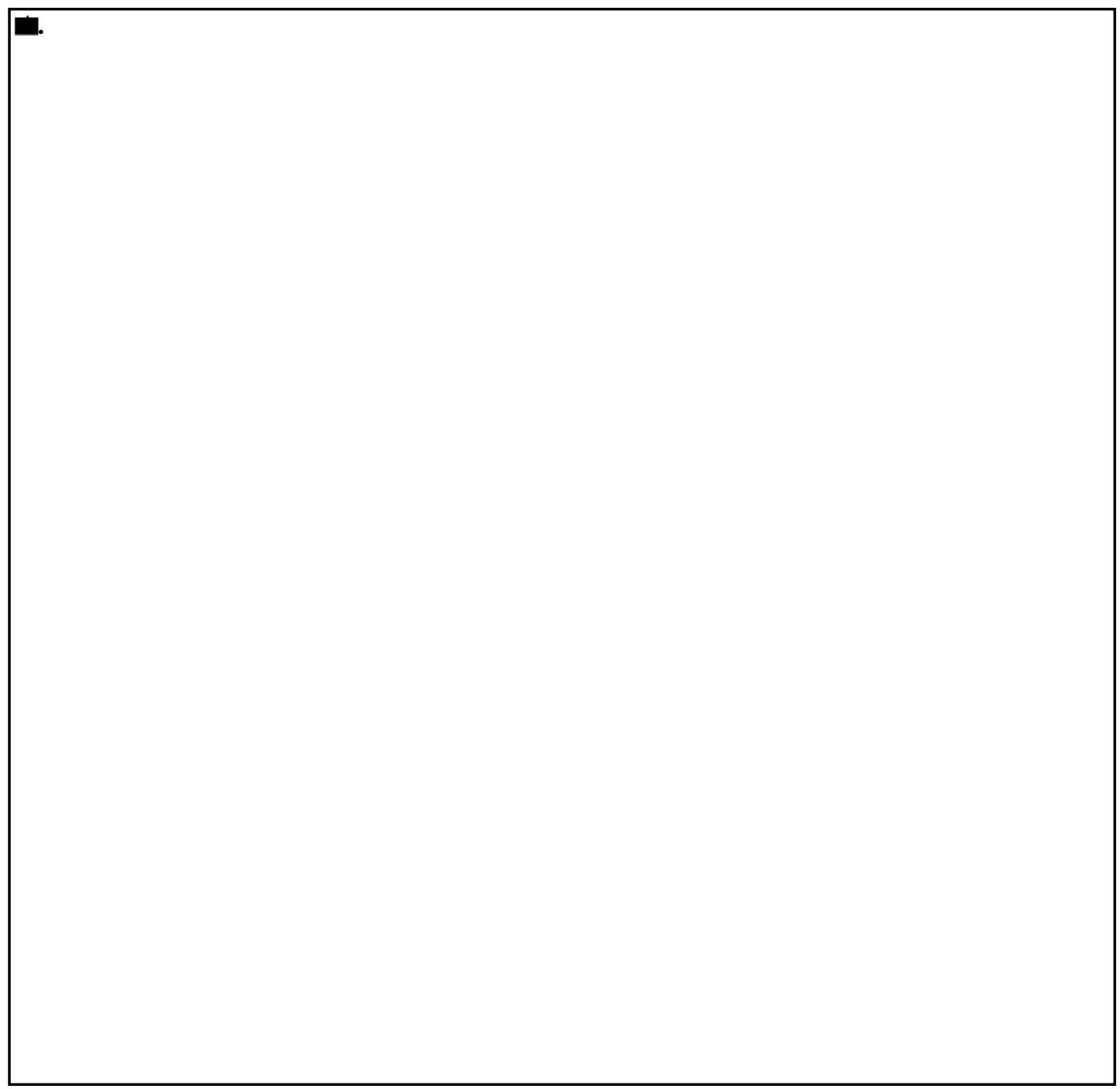

Fig. 4. Differential expression of mRNAs in IS patients $(n=10)$ and healthy controls $(n=10)$. (A) Heatmap of 733 differentially expressed mRNAs between the two groups. (B) Scatter plot of differential mRNA expression. X-axis: Test, Y-axis: Control. (C) Volcano plot of differential mRNA expression. X-axis: $\log _{2}$ FC (Test vs Control), Y-axis: $-\log _{10}$ (P value) for each probe. Each mRNA is represented by a dot, up-regulated as red dots $(\mathrm{FC} \geq 2, \mathrm{P}<0.05, \mathrm{FDR}<0.05)$ and down-regulated as green dots $(\mathrm{FC} \leq-2, \mathrm{P}<0.05, \mathrm{FDR}<0.05)$. The black dots in the center area represent the remaining $\operatorname{lncRNAs}(-2<\mathrm{FC}<2, \mathrm{P}<0.05, \mathrm{FDR}<0.05)$.

included to enrich GO functions (Fig. 5A-F) and KEGG pathways (Fig. 5G, H) by using DAVID bioinformatics online tools. Genes related to the up-/down-regulated mRNAs probably affected the IS process through significant GO categories or KEGG pathway $(P<0.05$, that is $-\log _{10} P$-value $>1.3$ ) (Table S5).

\section{LncRNA qRT-PCR validation and ROC curves}

At the first stage, nine differentially expressed lncRNAs were selected based on the criteria of absolute $\mathrm{FC} \geq 2, P<0.05$, and FDR $<0.05$ (sorting by FC, $P$, and FDR with consideration of RNA source and length). qRT-PCR analysis was performed to determine the expression level of these lncRNAs obtained from peripheral blood samples of patients with IS $(n=10)$ and healthy controls $(n=10)$ (Fig. 6A). The significantly and differentially up-regulated lncRNAs were ENST00000467369 (alias name: HCP5; $P=0.005, \mathrm{FC}=2.667$ ), ENST00000568297 (alias name: RP11-386M24.6; $P=0.036, \mathrm{FC}=7.868$ ), ENST00000568243

\section{KARGER}




\section{Cellular Physiology Cell Physiol Biochem 2018;50:2157-2175

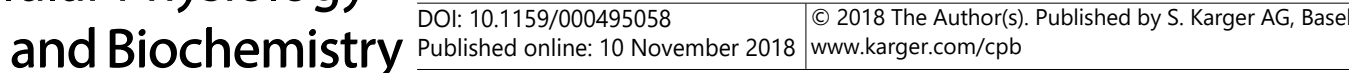 \\ Guo et al.: LncRNA Biomarkers and Networks in Chinese IS Patients}

Table 4. Top 25 of differently expressed mRNAs in IS patients compared to healthy controls (sorted by $|\mathrm{FC}|$ ). $|\mathrm{FC}|$, absolute fold change; FDR, false discovery rate

\begin{tabular}{|c|c|c|c|c|c|c|c|c|c|}
\hline \multirow{2}{*}{$\begin{array}{l}\text { Up-regulated mRNAs } \\
\text { Segname }\end{array}$} & \multicolumn{9}{|c|}{ Down-regulated mRNAs } \\
\hline & Gene Symbol & $|\mathrm{FC}|$ & $\mathrm{P}$ & FDR & Seqname & Gene Symbol & $|\mathrm{FC}|$ & $\mathrm{P}$ & FDR \\
\hline NM_006865 & LILRA3 & 20.570 & 0.005 & 0.031 & uc009vmq.3 & $\operatorname{mir}-34$ & 9.228 & $<0.001$ & 0.006 \\
\hline NM_002125 & HLA-DRB5 & 20.143 & 0.007 & 0.039 & NM_001753 & CAV1 & 8.685 & $<0.001$ & 0.004 \\
\hline NM_001099293 & KIF4B & 10.693 & $<0.001$ & 0.001 & NM_017805 & RASIP1 & 7.273 & 0.004 & 0.027 \\
\hline ENST00000589616 & LPHN1 & 8.814 & $<0.001$ & 0.006 & NM_001178139 & TFDP2 & 6.669 & $<0.001$ & 0.005 \\
\hline NM_152402 & TRAM1L1 & 6.509 & $<0.001$ & 0.006 & NM_019008 & MIEF1 & 6.647 & $<0.001$ & 0.001 \\
\hline NM_001925 & DEFA4 & 5.763 & 0.001 & 0.013 & NM_001304388 & GOLGA6L2 & 6.570 & 0.001 & 0.011 \\
\hline NM_031475 & ESPN & 5.611 & $<0.001$ & 0.002 & NM_145804 & АВTB2 & 6.495 & $<0.001$ & $<0.001$ \\
\hline NM_001911 & CTSG & 5.125 & 0.003 & 0.024 & NM_001282477 & TMEM210 & 6.403 & $<0.001$ & 0.004 \\
\hline NM_145286 & STOML3 & 4.649 & $<0.001$ & 0.005 & NM_006404 & PROCR & 6.231 & $<0.001$ & 0.006 \\
\hline NM_153269 & C20orf96 & 4.480 & $<0.001$ & 0.004 & NM_001105247 & ARMC5 & 5.765 & 0.004 & 0.027 \\
\hline NM_001008784 & CD200R1L & 4.358 & $<0.001$ & 0.005 & NM_012232 & PTRF & 5.571 & 0.003 & 0.023 \\
\hline NM_021069 & SORBS2 & 4.222 & 0.009 & 0.045 & NM_003881 & WISP2 & 5.531 & $<0.001$ & 0.003 \\
\hline NM_020747 & ZNF608 & 4.016 & $<0.001$ & 0.007 & NM_002632 & PGF & 5.381 & 0.001 & 0.008 \\
\hline NM_001136493 & MFSD2A & 4.011 & $<0.001$ & 0.003 & NM_022475 & HHIP & 5.300 & 0.006 & 0.036 \\
\hline NM_138787 & C11orf74 & 3.977 & 0.010 & 0.04 & NM_000424 & KRT5 & 5.175 & 0.001 & 0.013 \\
\hline NM_144973 & DENND5B & 3.772 & $<0.001$ & 0.002 & NM_017950 & CCDC40 & 5.108 & $<0.001$ & 0.004 \\
\hline NM_002237 & KCNG1 & 3.735 & 0.007 & 0.039 & NM_017659 & QPCTL & 4.902 & $<0.001$ & $<0.001$ \\
\hline NM_006235 & POU2AF1 & 3.631 & 0.001 & 0.013 & NM_004995 & MMP14 & 4.769 & $<0.001$ & 0.001 \\
\hline NM_030764 & FCRL2 & 3.514 & $<0.001$ & 0.006 & NM_178510 & ANKK1 & 4.657 & $<0.001$ & 0.004 \\
\hline NM_013363 & PCOLCE2 & 3.507 & 0.001 & 0.012 & NM_001077693 & ECSCR & 4.600 & 0.002 & 0.017 \\
\hline NM_004084 & DEFA1 & 3.474 & 0.001 & 0.014 & NM_001101401 & SBK2 & 4.569 & 0.003 & 0.023 \\
\hline NM_002343 & LTF & 3.456 & 0.003 & 0.023 & uc022bgl1 & AX748309 & 4.563 & $<0.001$ & 0.006 \\
\hline NM_001816 & CEACAM8 & 3.394 & 0.001 & 0.009 & uc003wfu.3 & AF035281 & 4.555 & 0.003 & 0.024 \\
\hline NM_001126128 & PROK2 & 3.386 & $<0.001$ & 0.004 & ENST00000529166 & NPIPA2 & 4.549 & 0.001 & 0.009 \\
\hline NM_001098833 & ATXN7L3 & 3.368 & $<0.001$ & $<0.001$ & NM_001039840 & CHIC1 & 4.502 & $<0.001$ & 0.003 \\
\hline
\end{tabular}

(alias name: RP11-408H20.1; $P<0.001, \mathrm{FC}=2.395$ ), and NR_046084 (alias name: SH3BP5$A S 1 ; P<0.0001, \mathrm{FC}=2.175)$. At the second stage, we expanded samples to validate the four significant lncRNAs by using 50 patients with IS and 50 controls. ENST00000568297 $(P=$ $0.025, \mathrm{FC}=2.965)$, ENST00000568243 $(P=0.017, \mathrm{FC}=1.302)$, and NR_046084 $(P=0.004$, $\mathrm{FC}=1.902)$ were significantly up-regulated in patients with IS compared with controls (Fig. 6B, C, D). The qRT-PCR results of these three IncRNAs, but not ENST00000467369 ( $P=$ $0.414, \mathrm{FC}=-1.268$ ), are consistent with the microarray findings. We expanded the sample size to further validate the up-regulation of ENST00000568297 $(P<0.0001, \mathrm{FC}=7.300)$, ENST00000568243 $(P<0.0001, \mathrm{FC}=1.841)$, and NR_046084 $(P<0.0001, \mathrm{FC}=2.265)$ in patients with IS compared with controls (Fig. 6E, F, G). These results were also consistent with the microarray analysis. Further, relative expression levels of the three significantly expressed lncRNAs were analyzed in sex subgroups (Fig. S1). These lncRNAs were also significantly up-regulated in male/female IS cases compared with male/female controls (ENST00000568297: $P=0.0006 / P=0.0005, \mathrm{FC}=6.98 / \mathrm{FC}=5.32 ; E N S T 00000568243: P<$ $0.0001 / P<0.0001, \mathrm{FC}=3.37 / \mathrm{FC}=3.36 ; N R \_046084: P<0.0001 / P=0.0132, \mathrm{FC}=2.46 / \mathrm{FC}=$ 1.87). Descriptions of the three significantly expressed lncRNAs are shown in Table 5.

As shown in Fig. 7A, B, and C, the ROC analysis indicated that the AUC values of ENST00000568297 (Sp = 63.6\%, Se = 64.8\%), ENST00000568243 ( $\mathrm{Sp}=69.5 \%$, Se = 70.5\%), and NR_046084 (Sp $=69.2 \%, \mathrm{Se}=61.5 \%)$ were $0.733,0.743$, and 0.690, respectively. The AUCs of the combined ROC curves of ENST00000568297 and ENST00000568243 (Fig. 7D); ENST00000568297 and NR_046084 (Fig. 7E); ENST00000568243 and NR_046084 (Fig. 7F); and ENST00000568297, ENST00000568243, and NR_046084 (Fig. 7G) were 0.776, 0.719, 0.787 , and 0.843 , respectively. Finally, the specificity $(80.0 \%)$ and sensitivity $(82.8 \%)$ were significantly improved; hence, the combination of the three whole lncRNAs was employed for IS diagnosis.

In addition, for the three validated IncRNAs that were up-regulated in IS patients compared to controls, correlation analysis with common clinical parameters (e.g. blood pressure, blood lipids, blood glucose) was performed in overall, male, and female samples. Most clinical parameters, including age, SBP, diastolic blood pressure, total cholesterol, triglyceride, high-density lipoprotein, low-density lipoprotein, and glucose, were not significantly correlated with expression of the three lncRNAs $(P>0.05)$. Nevertheless, some significant correlations were found. Details are described in Table S6. 


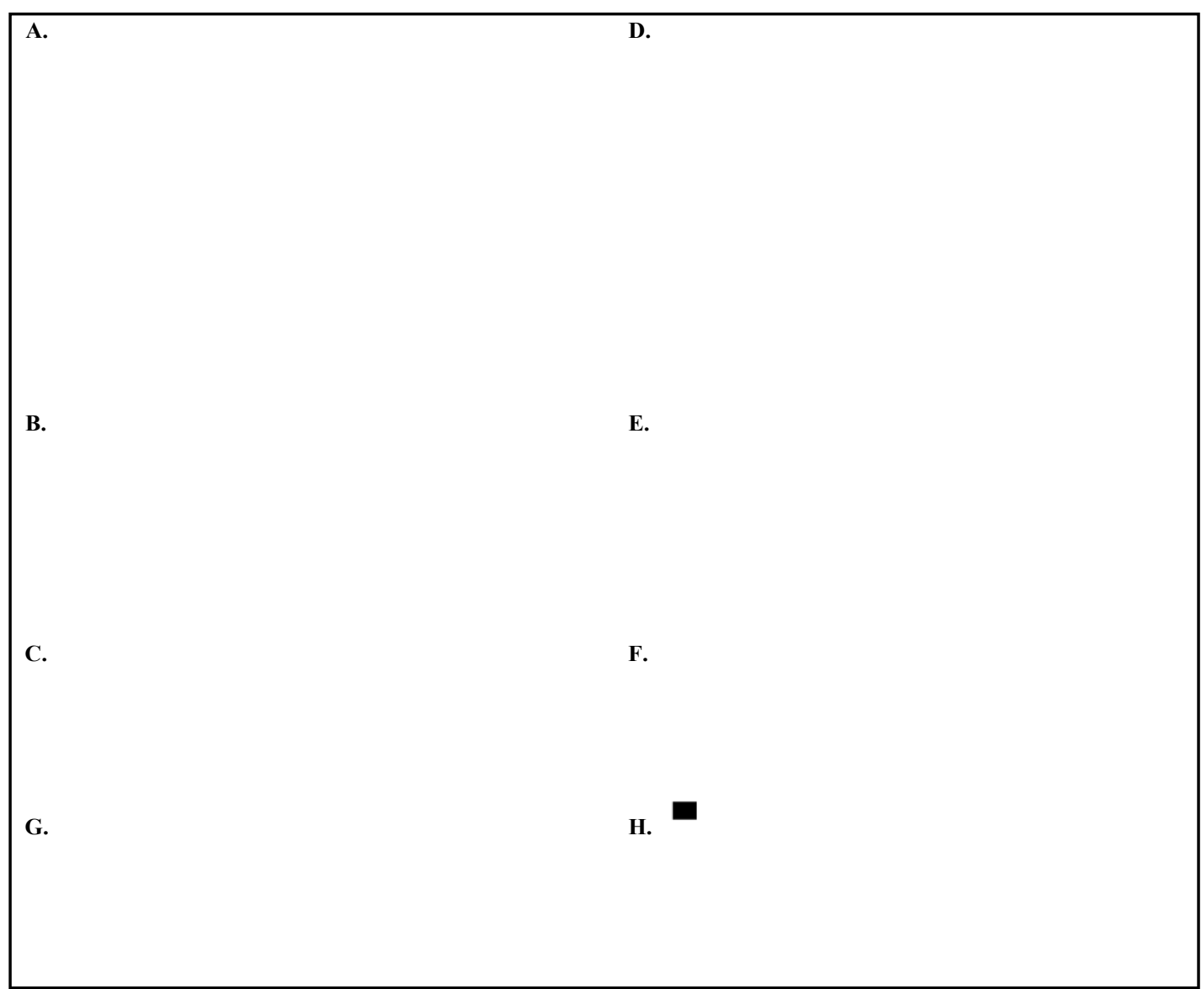

Fig. 5. Functional annotation of 249 up-regulated and 484 down-regulated genes from microarray. (A) Enriched GO terms $\left(-\log _{10} \mathrm{P}\right.$ value $\left.>1.3\right)$ related to BP of up-regulated mRNAs. (B) Enriched GO terms ($\log _{10} \mathrm{P}$ value $\left.>1.3\right)$ related to CC of up-regulated mRNAs. (C) Enriched GO terms $\left(-\log _{10} \mathrm{P}\right.$ value $\left.>1.3\right)$ related to MF of up-regulated mRNAs. (D) Enriched GO terms (- $\log _{10} \mathrm{P}$ value $\left.>1.3\right)$ related to $\mathrm{BP}$ of down-regulated mRNAs. (E) Enriched GO (- $\log _{10}$ P value $\left.>1.3\right)$ terms related to CC of down-regulated mRNAs. (F) Enriched GO terms $\left(-\log _{10}\right.$ P value $\left.>1.3\right)$ related to MF of down-regulated mRNAs. (G) Enriched KEGG pathways $(\mathrm{P}<0.05)$ of up-regulated mRNAs. $(\mathrm{H})$ Enriched KEGG pathways $(\mathrm{P}<0.05)$ of down-regulated mRNAs.

\section{Construction of CNC co-expression network}

We constructed a co-expression network consisting of differentially expressed lncRNAs and their corresponding mRNAs (Section 3.2) to determine their potential functions in IS progression. The CNC network contains 679 lncRNAs (165 up-regulated and 514 downregulated) and 561 mRNAs (136 up-regulated and 425 down-regulated). Fig. S2 and S3 show up-regulated CNC network and down-regulated CNC network, respectively. Fig. 8 shows the targeted CNC network of the three significantly expressed IncRNAs (ENST00000568297, ENST00000568243, and NR_046084). We performed GO functional annotation with mRNAs related to up-regulated IncRNAs within the CNC network. GO terms such as "regulation of branching morphogenesis of a nerve" and "positive regulation of dopamine receptor signaling pathway" were enriched. The mRNAs that interacted with ENST00000568297, ENST00000568243, or NR_046084 significantly enriched several GO terms (Table S7). 


\section{Cellular Physiology Cell Physiol Biochem 2018;50:2157-2175 and Biochemistry \begin{tabular}{c|c|c|} 
DOl: 10.1159/000495058 & $\begin{array}{l}\text { O } 2018 \text { The Author(s). Published by S. Karger AG, Basel } \\
\text { www.karger.com/cpb }\end{array}$
\end{tabular}

Fig. 6. Relative expression level of (A) the 9 differentially up-regulated IncRNAs; (B, E) ENST00000568297; (C, F) ENST00000568243; (D, G) NR_046084. * represents $\mathrm{P}<0.05$, ** represents $\mathrm{P}<0.01$, $* * * \quad$ represents $\mathrm{P}<0.001$, **** represents $\mathrm{P}<0.0001$. (Notice: at stage one, only ENST00000568243 was performed with the ABI StepOnePlus Real-Time PCR System using stage two/three methods).

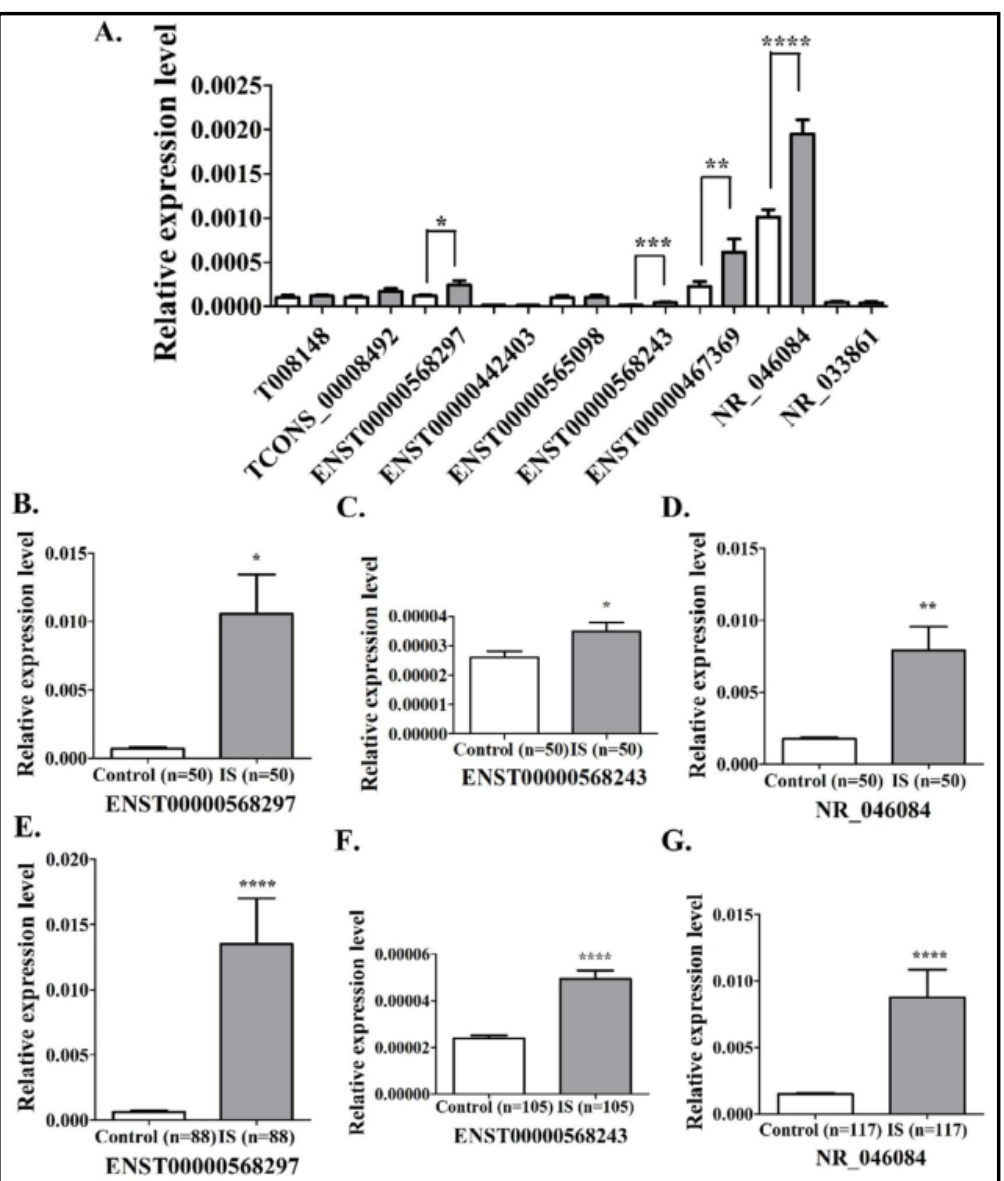

Table 5. Information of the 3 differentially expressed lncRNAs in qRT-PCR. FDR, false discovery rate; $|F C|$, absolute fold change; Reg, regulation; Chr, chromosome

\begin{tabular}{lccccccccccc}
\hline Name & Gene Symbol & Source & P & FDR & $\mid$ FC $\mid$ & Reg & Length & Chr & Strand & Start & End \\
\hline ENST00000568297 & RP11-386M24.6 & GENCODE & 0.011 & 0.047 & 7.868 & up & 374 & chr15 & - & 93138789 & 93139692 \\
ENST00000568243 & RP11-408H20.1 & GENCODE & 0.007 & 0.034 & 5.641 & up & 554 & chr18 & - & 28638805 & 28639359 \\
NR_046084 & SH3BP5-AS1 & RefSeq & $<0.001$ & 0.003 & 2.175 & up & 5592 & chr3 & + & 15295690 & 15306005 \\
\hline
\end{tabular}

Construction of IncRNA-miRNA-mRNA regulatory network

Using the three validated distinctly up-regulated IncRNAs (ENST00000568297, ENST00000568243, and NR_046084), we constructed a comprehensive IncRNA-miRNAmRNA regulatory network to interact with 81 predicted miRNAs and 7, 312 targeted mRNAs. Fig. S4 shows the three lncRNAs, 81 miRNAs, and 824 mRNAs (each of which interacted with at least five miRNAs). Specifically, IncRNA-ENST00000568297 targeted to 33 miRNAs, IncRNAENST00000568243 targeted to 21 miRNAs, and IncRNA-NR_046084 targeted to 28 miRNAs. Furthermore, THBS1 was regulated by 10 miRNAs, AKT2 was regulated by 9 miRNAs, PTEN was regulated by 7 miRNAs, $A K T 3$ and MAPK1 were regulated by 5 miRNAs, and SMAD2 and SMAD3 were regulated by 6 miRNAs. The KEGG pathways (Fig. 9) were enriched with 824 mRNAs (each of which interacted with at least five miRNAs) in the lncRNA-miRNA-mRNA regulatory network. A total of 43 significant pathways $(P<0.01)$ were enriched using DAVID Bioinformatics Resources v6.8 online tools and included six IS-related pathways (Table 6): "PI3K-Akt signaling pathway", "p53 signaling pathway", "AMPK signaling pathway", "MAPK signaling pathway", "Wnt signaling pathway", and "TGF-beta signaling pathway". As shown in Fig. S5 and Table S9, the IncRNA-miRNA-mRNA-pathway regulatory network revealed indirect relations between the three distinguished lncRNAs and the six important KEGG pathways. 
Fig. 7. ROC curve of (A) ENST 00000568297 $(\mathrm{P}<0.0001, \quad \mathrm{AUC}=$ $0.733, \mathrm{Sp}=63.6 \%, \mathrm{Se}=$ $64.8 \%$, cut-off $=0.001$ ); (B) ENST00000568243 $(\mathrm{P}<0.0001, \quad \mathrm{AUC}=$ $0.743, \mathrm{Sp}=69.5 \%$, $\mathrm{Se}=70.5 \%$, cutoff = 0.00003); (C) NR_046084 $(\mathrm{P}<0.0001$, $\mathrm{AUC}=0.690, \mathrm{Sp}=$ $69.2 \%$, Se = 61.5\%, cut-off = 0.002); (D) ENST 00000568297 a $\mathrm{n}$

ENST 00000568243 $(\mathrm{P}<0.0001$, AUC = $0.776, \mathrm{Sp}=75.0 \%, \mathrm{Se}=$ $75.9 \%$, cut-off $=0.489$ ); (E) ENST00000568297 and NR_046084 $(\mathrm{P}<0.0001, \quad \mathrm{AUC}=$ $0.719, \mathrm{Sp}=67.5 \%, \mathrm{Se}=$ $70.7 \%$, cut-off $=0.473$ ); (F) ENST00000568243 and NR_046084
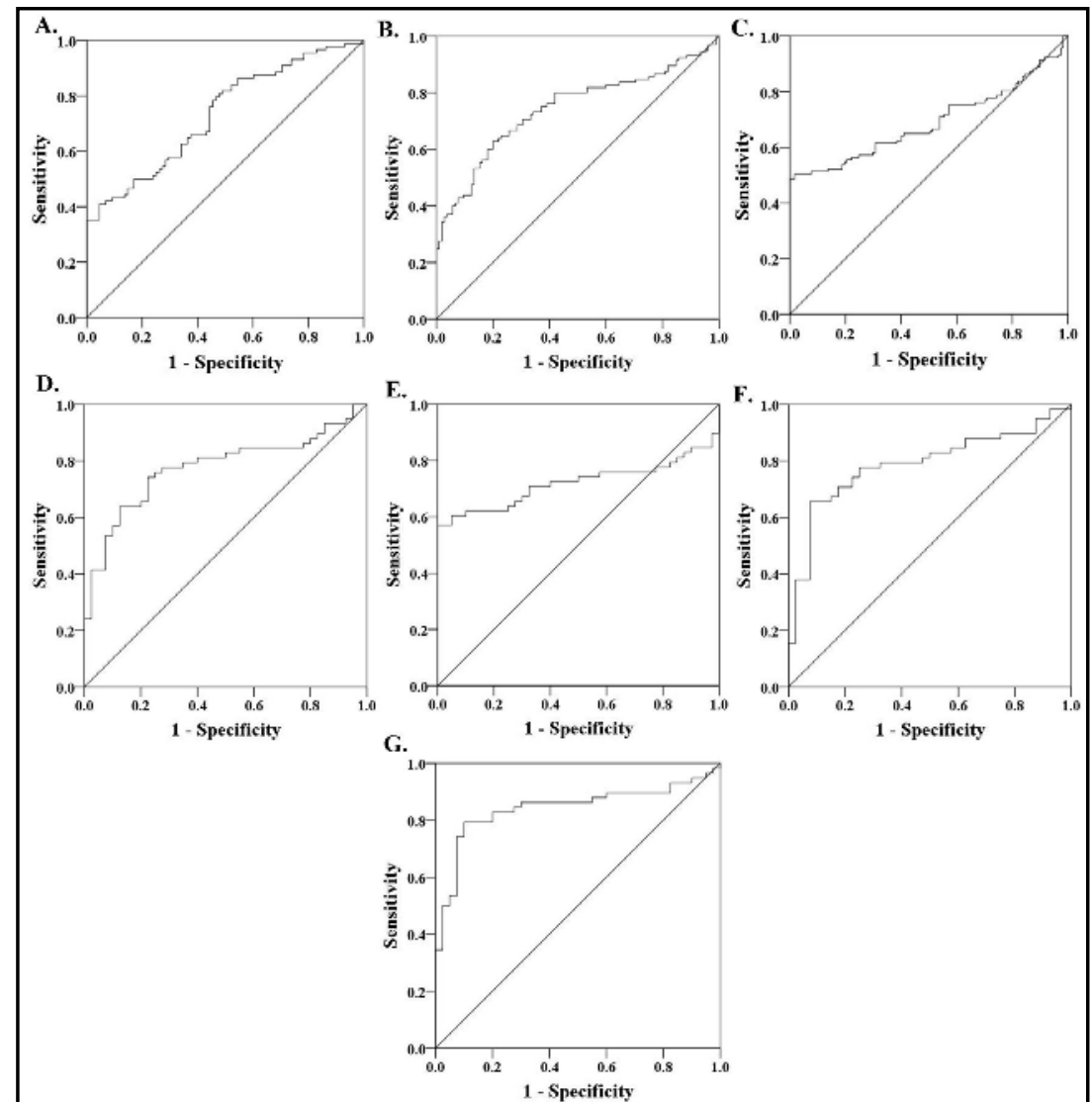

$(\mathrm{P}<0.0001, \quad$ AUC $=$

$0.787, \mathrm{Sp}=75.0 \%$, Se $=77.6 \%$, cut-off $=0.509) ;(G)$ ENST00000568297, ENST00000568243, and NR_046084 $(\mathrm{P}<0.0001, \mathrm{AUC}=0.843, \mathrm{Sp}=80.0 \%, \mathrm{Se}=82.8 \%$, cut-off $=0.466)$.

Fig. 8. CNC network with PCC $\geq 0.90$ and $\mathrm{P}<0.05$. Triangles represent up-regulated IncRNAs; circles represent upregulated mRNAs; IncRNAs and mRNAs in the co-expressed network are expressed in the same direction. Details are presented in Table S8.

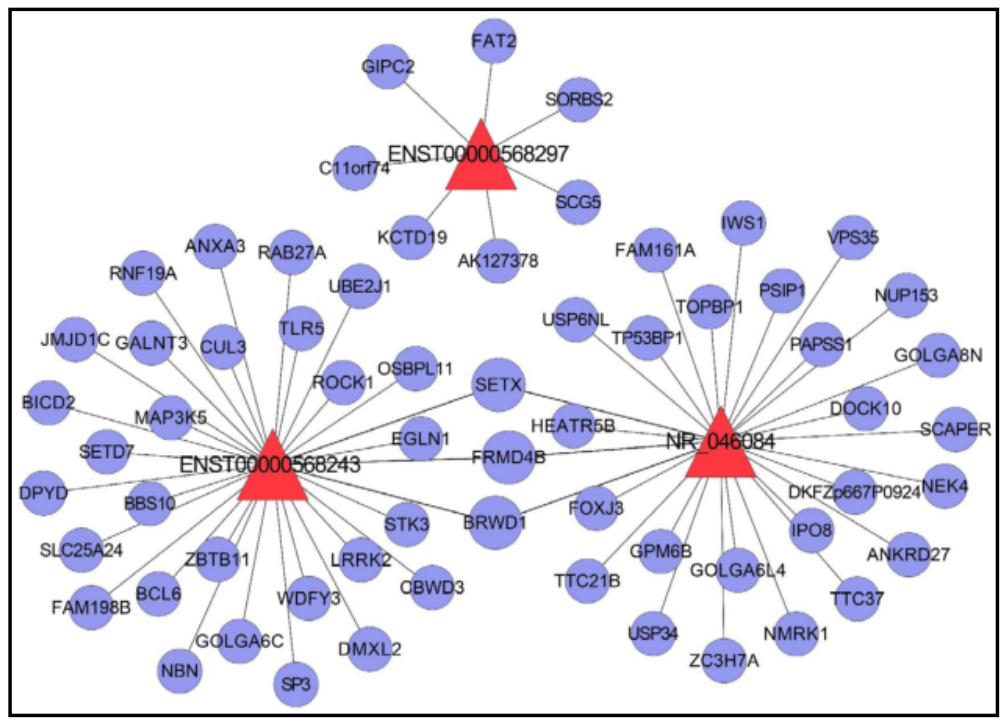


Fig. 9. Enriched KEGG pathways $(\mathrm{P}<0.01)$ of the 824 genes from the IncRNA-miRNA-mRNA regulation network.

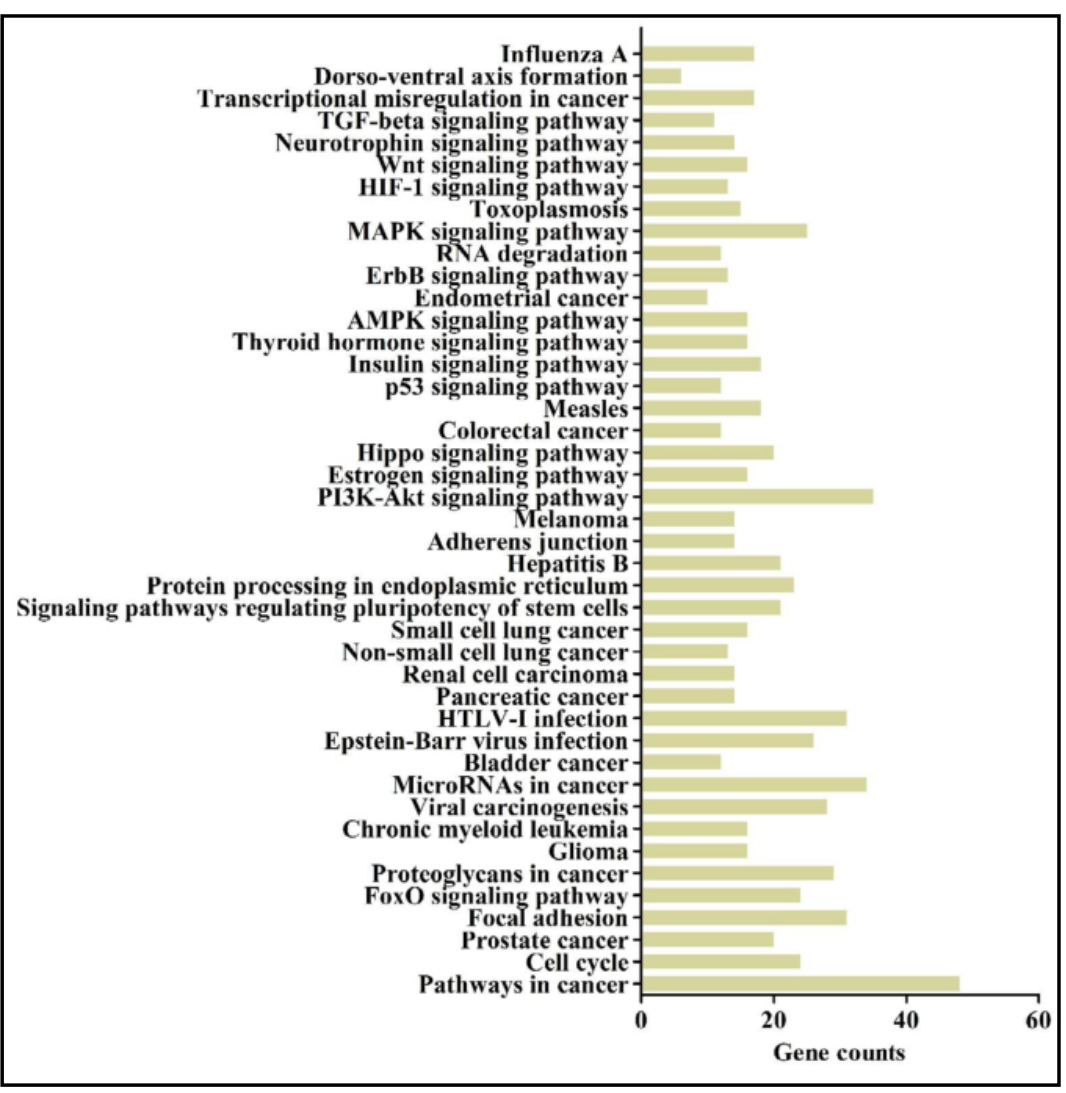

Table 6. Information of the 6 KEGG pathways. N, number of genes; FE, fold enrichment; FDR, false discovery rate

\begin{tabular}{|c|c|c|c|c|c|c|c|c|c|c|}
\hline Term & $\mathrm{N}$ & $\%$ & $\mathrm{P}$ & List Total & Pop Hits & Pop Total & FE & Bonferroni & Benjamini & FDR \\
\hline $\begin{array}{l}\text { PI3K-Akt signaling pathway } \\
\text { (hsa04151) }\end{array}$ & 35 & $\begin{array}{l}4.3 \\
16\end{array}$ & $<0.001$ & 335 & 345 & 6910 & 2.093 & 0.012 & 0.001 & 0.064 \\
\hline Genes & \multicolumn{10}{|c|}{$\begin{array}{l}\text { HSP90AB1, MCL1, GRB2, TNC, PTEN, IGF1R, PPP2CA, SOS1, CREB3L2, PRKAA1, THBS1, MYC, INSR, AKT3, FN1, AKT2, MET, CDK6, CDK4, YWHAE, BCL2L11, } \\
\text { MAPK1, CCND1, LAMA4, HSP90B1, CDKN1A, YWHAG, CDKN1B, ITGA6, CCND2, GSK3B, VEGFA, JAK1, MDM2, LAMC1 }\end{array}$} \\
\hline $\begin{array}{l}\text { p53 signaling pathway } \\
\text { (hsa04115) }\end{array}$ & 12 & $\begin{array}{l}1.4 \\
80\end{array}$ & $<0.001$ & 335 & 67 & 6910 & 3.694 & 0.074 & 0.003 & 0.413 \\
\hline Genes & \multicolumn{10}{|c|}{ CCNB1, CDKN1A, CASP3, CCND1, CCND2, ZMAT3, MDM2, CDK6, THBS1, CDK4, PTEN, SESN3 } \\
\hline $\begin{array}{l}\text { AMPK signaling pathway } \\
\text { (hsa04152) }\end{array}$ & 16 & $\begin{array}{l}1.9 \\
73\end{array}$ & 0.001 & 335 & 122 & 6910 & 2.705 & 0.162 & 0.006 & 0.951 \\
\hline Genes & \multicolumn{10}{|c|}{ IRS4, RAB2A, IGF1R, CCND1, PPP2CA, PRKAB2, FASN, RAB14, CREB3L2, ADIPOR2, ELAVL1, PRKAA1, EEF2, INSR, AKT3, AKT2 } \\
\hline $\begin{array}{l}\text { MAPK signaling pathway } \\
\text { (hsa04010) }\end{array}$ & 25 & $\begin{array}{l}3.0 \\
83\end{array}$ & 0.001 & 335 & 255 & 6910 & 2.022 & 0.261 & 0.009 & 1.623 \\
\hline Genes & \multicolumn{10}{|c|}{$\begin{array}{l}\text { GRB2, PPM1A, MKNK2, SRF, CDC42, CASP3, PAK2, ELK4, MAP3K2, SOS1, PRKACB, MYC, AKT3, HSPA8, AKT2, TAOK2, TAOK1, STK4, TAB2, FLNA, MAPK1, CRKL, } \\
\text { JUN, STMN1, DUSP7 }\end{array}$} \\
\hline $\begin{array}{l}\text { Wnt signaling pathway } \\
\text { (hsa04310) }\end{array}$ & 16 & $\begin{array}{l}1.9 \\
73\end{array}$ & 0.003 & $\begin{array}{c}335 \\
-128\end{array}$ & 138 & 6910 & $\begin{array}{r}2.392 \\
-2 N K 1 F\end{array}$ & 0.462 & 0.017 & 3.304 \\
\hline Genes & \multicolumn{10}{|c|}{ CSNK1A1, DVL3, TBL1XR1, FZD3, FZD6, CTNNB1, CCND1, EP300, CSNK2A1, CSNK1E, CCND2, JUN, GSK3B, PRKACB, MYC, FOSL1 } \\
\hline $\begin{array}{l}\text { TGF-beta signaling pathway } \\
\text { (hsa04350) }\end{array}$ & 11 & $\begin{array}{l}1.3 \\
56\end{array}$ & 0.007 & 335 & 84 & 6910 & 2.701 & 0.810 & 0.043 & 8.592 \\
\hline Genes & \multicolumn{10}{|c|}{ MAPK1, ACVR2B, EP300, SP1, SMAD7, PPP2CA, BMPR2, SMAD3, SMAD2, THBS1, MYC } \\
\hline
\end{tabular}

\section{Discussion}

This study identified human IncRNA and mRNA profiles of IS, determined three IncRNAs as potential diagnostic biomarkers of IS, and predicted the potential functions on IS pathogenesis through bioinformatics analysis. To our knowledge, this study is the first to investigate the IncRNA expression profile of IS in Chinese patients by using human peripheral blood samples. LncRNA-ENST00000568297, IncRNA-ENST00000568243, and lncRNA-NR_046084 were identified as potential diagnostic biomarkers of IS. The up-/downregulated $\mathrm{CNC}$ networks revealed the interactions of IncRNAs and mRNAs. GO functional annotation of the mRNAs that interacted with ENST00000568243 enriched the following: "regulation of neuron death", "activation of MAPKK activity", and "positive regulation of 


\section{Cellular Physiology Cell Physiol Biochem 2018;50:2157-2175

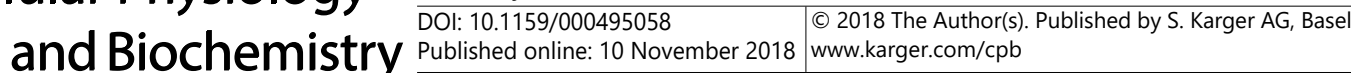 \\ Guo et al.: LncRNA Biomarkers and Networks in Chinese IS Patients}

apoptotic process", which are related to biological process (BP); and "protein kinase activity", which is related to molecular function (MF). In the IncRNA-miRNA-mRNA regulatory network, KEGG pathway analysis indicated that lncRNAs may regulate relative mRNAs via "PI3K-Akt signaling pathway", "p53 signaling pathway", "AMPK signaling pathway", "MAPK signaling pathway", "Wnt signaling pathway", and "TGF-beta signaling pathway" to perform functions.

LncRNAs are dysregulated in several diseases, such as obesity, ischemic heart failure, moyamoya disease, and stroke [12-15]. Dykstra-Aiello explored the lncRNA expression profile of IS and stroke risk loci by using human peripheral blood obtained from American and Canadian subjects; the results identified 299 and 97 differentially expressed lncRNAs in males and females, respectively $(P<0.005,|\mathrm{FC}|>1.2)$ [12]. Affymetrix GeneChip Human Transcriptome 2.0 arrays ( $>40,000$ lncRNAs and $>245,000$ coding transcripts) were used to detect significantly altered lncRNAs in the Dykstra-Aiello study; meanwhile, we used Arraystar Human LncRNA Microarray v4.0 (40, 173 lncRNAs and 20, 730 coding transcripts) in the present study. However, Dykstra-Aiello performed a microarray analysis without qRT-PCR validation, and therefore the microarray analysis may yield false-positive results. Experimental techniques such as qRT-PCR are essential to validate the microarray result. We identified differentially expressed lncRNAs according to strict and common criteria of $|\mathrm{FC}|$ $\geq 2, P<0.05$, and FDR $<0.05$. Recently, Deng identified novel lncRNA diagnostic biomarkers for acute ischemic stroke (AIS) in peripheral blood mononuclear cells (PBMCs) using Human LncRNA Array v4 (CapitalBio Technology, China) [16]. A total of 70 up-regulated and 128 down-regulated IncRNAs were identified by microarray to be differentially expressed in five AIS patients compared to five controls with a criterion of $|\mathrm{FC}|>2$ and $P<0.05$. Deng included a total of 206 IS and 179 healthy control samples for the study and finally determined three lncRNAs (linc-DHFRL1-4, SNHG15, and linc-FAM98A-3) as IS diagnostic biomarkers. By comparison, although relatively fewer samples were included in the current study, potential lncRNA diagnostic markers were also established and further preliminary bioinformatics analysis was performed to explore potential functions of the three lncRNAs in IS pathology.

Circulating lncRNAs are a novel category of promising biomarkers and play key roles in screening, diagnosis, process, treatment, and prognosis of many diseases [17-19]. Serum IncRNA-RP11-445H22.4 has been confirmed as a biomarker for detecting breast cancer and exhibited AUC of 0.904 ( $\mathrm{Sp}=74 \%$, Se $=92 \%$ ) [20]. Ying Zhang revealed IncRNA-ZFAS1 and IncRNA-CDR1AS as novel predictors for acute myocardial infarction (AMI) in human whole blood; the AUC was 0.664 or 0.671 when AMI was diagnosed with IncRNA-ZFAS1 or IncRNA-CDR1AS alone and increased to 0.691 when the two IncRNAs were combined [21]. We first evaluated the diagnostic value of IncRNAs in human peripheral blood samples for IS based on IncRNA microarray screening and qRT-PCR validations. The AUCs were as follows: ENST00000568297 alone, 0.733; ENST00000568243 alone, 0.743; NR_046084 alone, 0.690; ENST00000568297 and ENST00000568243, 0.776; ENST00000568297 and NR_046084, 0.719; ENST00000568243 and NR_046084, 0.787; combination of all, 0.843 . The AUC of the combination of the three lncRNAs is significantly increased compared with that of each single lncRNA. At the optimal cut-off points, the sensitivity values of ENST00000568297, ENST00000568243, NR_046084, ENST00000568297 and ENST00000568243, ENST00000568297 and NR_046084, ENST00000568243 and NR_046084, and the combination of all are $64.8 \%, 70.5 \%, 61.5 \%, 75.9 \%, 75.9 \%, 70.7 \%$, and $82.8 \%$, respectively; the specificity values are $63.6 \%, 69.5 \%, 69.2 \%, 75.0 \%, 67.5 \%, 75.0 \%$, and $80.0 \%$, respectively. The sensitivity (82.8\%) and specificity (80.0\%) of the combination of the three IncRNAs is significantly improved compared with single ENST00000568297 or single $N R$ 046084. This finding suggests that the combination of the three lncRNAs will provide higher diagnostic performance for IS diagnosis. Based on the correlation analysis these three potential IncRNA diagnostic biomarkers for IS were independent of some common clinical risk factors for IS, which may suggest the IncRNA biomarkers could be used for IS diagnosis independently. 


\section{Cellular Physiology Cell Physiol Biochem 2018;50:2157-2175

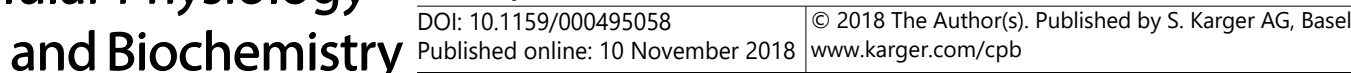 \\ Guo et al.: LncRNA Biomarkers and Networks in Chinese IS Patients}

Protein coding and non-coding RNAs regulate each other to perform their functions. For example, non-coding and coding RNAs connect to each other in the CNC network; as such, noncoding RNAs affect protein expression by regulating the effects of coding RNAs on diseases. Thus, gene functional annotation and pathway analyses are essential. Based on GO analysis, several gene functions related to BP, cellular composition (CC), and MF were enriched. With up-regulated mRNAs in the microarray, "positive regulation of c-Jun N-terminal kinase (JNK) cascade" was enriched and regarded as a significant GO term related to BP. JNK has been reported to play a critical role in ischemic brain injury [22]. The up-regulated mRNAs in the microarray and mRNAs related to up-regulated lncRNAs within the CNC network both enriched the GO term "positive regulation of dopamine receptor signaling pathway", related to BP. An increased level of dopamine D2 receptor, which as a part of the dopamine receptor signaling pathway acts as a key target in the regulation of neuroinflammatory response, had a neuroprotective effect in OGD-treated astrocytes and MCAO-treated mice [23]. Focused on the three validated IncRNAs (ENST00000568297, ENST00000568243, and NR_046084), several GO categories that have been reported to be associated with the pathological mechanism of IS were enriched significantly. Interestingly, the category named "cellular response to oxidative stress" supported that IncRNAs participate in the process of oxidative stress in cells [24], probably through regulating mRNAs such as SLC25A24, LRRK2, and SETX. Kim reviewed and indicated that lncRNAs play important roles in cellular responses to oxidative stress; for example, cytoplasmic IncRNA LINK-A interacts with LRRK2 and was able to enhance phosphorylation of HIF- $1 \alpha$ at Ser797 in signal transduction under normoxic conditions [25]. In addition, the three lncRNAs-interacted mRNAs within the up-regulated CNC network could also enrich some other categories of importance, such as "MAPK cascade" and "protein phosphorylation". Apoptotic stress induced ASK1 (alias: MAP3K5) activation is promoted by Trx1siRNA, performing as ASK1/Trx1 binding complex, and ischemic reperfusion destroyed the complex and led to attenuation of the ASK1-JNK/p38 signaling pathway (part of the MAPK cascade) [26]. As well, protein phosphorylation plays key roles in MAPK signaling activation.

Analysis of the KEGG pathway related to 824 mRNAs (each of which interacted with $\geq$ 5 miRNAs, targeted to IncRNA-ENST00000568297, IncRNA-ENST00000568243, and IncRNA$N R \_046084$ ) in the IncRNA-miRNA-mRNA regulatory network enriched several interesting items. In particular, the following six stroke-related signaling pathways were outstanding: "PI3K-Akt signaling pathway", "p53 signaling pathway", "AMPK signaling pathway", "MAPK signaling pathway", "Wnt signaling pathway", and "TGF-beta signaling pathway". These pathways were enriched by some prominent mRNAs, such as AKT2, AKT3, MAPK1, PTEN, SMAD2, SMAD3, and THBS1, which are known to be associated with IS.

The MAPK signaling pathway has been implicated in inflammatory responses and cell apoptosis $[27,28]$. Anti-CKLF1 antibody inhibited neutrophil influx into the ischemic region via the MAPK signaling pathway, and the inhibition of $C K L F 1$ activity significantly protected against ischemia/reperfusion injury [29]. In rat brains, CBSA-PEG-TIIA-NPs (cationic bovine serum albumin-conjugated tanshinone IIA PEGylated nanoparticles) displayed distinguished neuroprotective effects on IS by modulating MAPK signaling pathways involved in the neuroinflammatory cascade [30]. Yaning Li reported the $S D F-1 \alpha$-CXCR4 interaction to activate $A K T, E R K$, and p38 MAPK signaling pathways in post-acute ischemic mice; $S D F-1 \alpha$ could promote neurogenesis and angiogenesis without eliciting inflammatory responses [31]. Lin revealed that the p38 MAPK/calpain 1 signaling pathway participated in endogenous neurorepair and spontaneous recovery after IS, mediated by $I L-17 A$ from astrocytes [32]. Both the MAPK and PI3K/Akt signaling pathways are involved in cerebral ischemic injury associated with TRPV4-induced apoptosis [33]. The PI3K/Akt/mTOR signaling pathway mediates ZSTK474 (a PI3K inhibitor) to inhibit inflammatory responses, reduce infarct volume, and exert neuroprotection in IS mice [34]. Akt phosphorylation was found to mediate the neuroprotective activity of melatonin, and melatonin inhibited p53 phosphorylation through the PI3K/Akt signaling pathway to increase neuronal survival [35]. The up- and down-stream components of PI3K/Akt signaling pathways, such as PTEN and mTOR, were 
also activated. TGF-beta acted as an anti-inflammatory factor. Myeloid TGF $\beta$ signaling has been suggested to be utilized for interference with stroke etiology-associated inflammation or vascular diseases [36]. The central intracellular mediator of TGF-beta signaling, namely, SMAD4, has been implicated in maintaining cerebrovascular integrity [37].

In the pathophysiology of stroke, p53 acts as master regulator in cell death [38]. Balaganapathy demonstrated that the Notch intracellular domain (NICD)-p53 interaction contributed to IS pathogenesis and enhanced the expression of pro-apoptotic genes following IS [39]. In rat cerebral cortical neurons, the ANXA1-p53 interaction in the nucleus was enhanced following oxygen-glucose deprivation-reoxygenation (OGD/R) and induced cell death [40]. The Wnt signaling pathway is involved in the development of the blood-brain barrier, contributed to stimulating neurogenesis, consolidating the blood-brain structure, and recovering cognitive brain functions after central nervous system injury [41-43]. Chang indicated that the Gpr124-Wnt signaling axis is essential for protecting blood-brain barrier function and vascular integrity [44]. In general, the AMPK signaling pathway is considered important in treatment of IS; metformin activated the AMPK signaling pathway to downregulate $I C A M-1$ expression, thereby effectively alleviating neutrophil infiltration to prevent brain injury induced by ischemia [45].

The regulatory mechanism between the upper coding and non-coding RNAs remains undetermined. We inferred that the three potential diagnostic biomarkers (ENST00000568297, ENST00000568243, NR_046084) participated in the pathophysiological processes of IS, possibly by regulating IS-related signaling pathways or pivotal miRNAs and mRNAs.

\section{Limitations}

MiRNAs included in the regulatory network were predicted by algorithmic model in comprehensive databases, and experimental validations in Han Chinese were performed well. We performed some of the basic bioinformatics analyses. The $\mathrm{CNC}$ co-expression network and the IncRNA-miRNA-mRNA regulatory network were constructed; however, further experiments (in vivo or in vitro) must be performed to validate the functions of the three lncRNAs. The three lncRNAs are potential biomarkers for diagnosis of general IS patients. Further analysis such as exploring the association of IS subtypes and the lncRNAs is required. Thus, we will explore the ceRNA mechanism of the IncRNA-miRNA-mRNA regulatory network by using experimental technologies, such as luciferase report gene, RNA RIP, and RNA pull down.

\section{Conclusion}

This study found that the lncRNA and mRNA expression profiles in human peripheral blood were altered after IS. Three IS-associated IncRNAs, namely, ENST00000568297, ENST00000568243, and NR_046084, were validated and identified as novel potential biomarkers for diagnosis of IS. These lncRNAs probably play important roles in IS pathophysiology by regulating IS-related pathways, mRNAs in the CNC co-expression network, or pivotal miRNAs and mRNAs within IncRNA-miRNA-mRNA regulatory networks.

\section{Acknowledgements}

This work is supported by the National Natural Science Foundation of China (No. 81860822, No. 81473670 and No. 81573756). 


\section{Cellular Physiology Cell Physiol Biochem 2018;50:2157-2175 and Biochemistry Published \begin{tabular}{l|l} 
DOI: 10.1159/000495058 & (c)18 The Author(s). Published by S. Karger AG, Basel \\
www.karger.com/cpb
\end{tabular}

\section{Disclosure Statement}

All authors declare no conflicts of interest.

\section{References}

1 Miller JB, Merck LH, Wira CR, Meurer WJ, Schrock JW, Nomura JT, Siket MS, Madsen TE, Wright DW, Panagos PD, Lewandowski C: The Advanced Reperfusion Era: Implications for Emergency Systems of Ischemic Stroke Care. Ann Emerg Med 2017;69:192-201.

-2 Feigin VL, Krishnamurthi RV, Parmar P, Norrving B, Mensah GA, Bennett DA, Barker-Collo S, Moran AE, Sacco RL, Truelsen T, Davis S, Pandian JD, Naghavi M, Forouzanfar MH, Nguyen G, Johnson CO, Vos T, Meretoja A, Murray CJ, Roth GA, Group GBDW, Group GBDSPE: Update on the Global Burden of Ischemic and Hemorrhagic Stroke in 1990-2013: The GBD 2013 Study. Neuroepidemiology 2015;45:161-176.

3 Feigin VL, Lawes CM, Bennett DA, Barker-Collo SL, Parag V: Worldwide stroke incidence and early case fatality reported in 56 population-based studies: a systematic review. Lancet Neurol 2009;8:355-369.

4 Achawanantakun R, Chen J, Sun Y, Zhang Y: LncRNA-ID: Long non-coding RNA IDentification using balanced random forests. Bioinformatics 2015;31:3897-3905.

5 Iwakiri J, Hamada M, Asai K: Bioinformatics tools for lncRNA research. Biochim Biophys Acta 2016;1859:23-30.

6 Yu B, Zhou S, Yi S, Gu X: The regulatory roles of non-coding RNAs in nerve injury and regeneration. Prog Neurobiol 2015;134:122-139.

7 Dharap A, Nakka VP, Vemuganti R: Effect of focal ischemia on long noncoding RNAs. Stroke 2012;43:28002802.

8 Wu Z, Wu P, Zuo X, Yu N, Qin Y, Xu Q He S, Cen B, Liao W, Ji A: LncRNA-N1LR Enhances Neuroprotection Against Ischemic Stroke Probably by Inhibiting p53 Phosphorylation. Mol Neurobiol 2016;10.1007/ s12035-016-0246-z.

-9 Dharap A, Pokrzywa C, Vemuganti R: Increased binding of stroke-induced long non-coding RNAs to the transcriptional corepressors Sin3A and coREST. ASN Neuro 2013;5:283-289.

10 Bhattarai S, Pontarelli F, Prendergast E, Dharap A: Discovery of novel stroke-responsive lncRNAs in the mouse cortex using genome-wide RNA-seq. Neurobiol Dis 2017;108:204-212.

11 Zhang J, Yuan L, Zhang X, Hamblin MH, Zhu T, Meng F, Li Y, Chen YE, Yin KJ: Altered long non-coding RNA transcriptomic profiles in brain microvascular endothelium after cerebral ischemia. Exp Neurol 2016;277:162-170.

12 Dykstra-Aiello C, Jickling GC, Ander BP, Shroff N, Zhan X, Liu D, Hull H, Orantia M, Stamova BS, Sharp FR: Altered Expression of Long Noncoding RNAs in Blood After Ischemic Stroke and Proximity to Putative Stroke Risk Loci. Stroke 2016;47:2896-2903.

13 Greco S, Zaccagnini G, Perfetti A, Fuschi P, Valaperta R, Voellenkle C, Castelvecchio S, Gaetano C, Finato N, Beltrami AP, Menicanti L, Martelli F: Long noncoding RNA dysregulation in ischemic heart failure. J Transl Med 2016;14:183.

-14 Sun J, Ruan Y, Wang M, Chen R, Yu N, Sun L, Liu T, Chen H: Differentially expressed circulating LncRNAs and mRNA identified by microarray analysis in obese patients. Sci Rep 2016;6:35421.

15 Wang W, Gao F, Zhao Z, Wang H, Zhang L, Zhang D, Zhang Y, Lan Q, Wang J, Zhao J: Integrated Analysis of LncRNA-mRNA Co-Expression Profiles in Patients with Moyamoya Disease. Sci Rep 2017;7:42421.

-16 Deng QW, Li S, Wang H, Sun HL, Zuo L, Gu ZT, Lu G, Sun CZ, Zhang HQ, Yan FL: Differential long noncoding RNA expressions in peripheral blood mononuclear cells for detection of acute ischemic stroke. Clin Sci (Lond) 2018;10.1042/CS20180411.

17 Schmitt AM, Chang HY: Long Noncoding RNAs in Cancer Pathways. Cancer Cell 2016;29:452-463.

18 Quan Z, Zheng D, Qing H: Regulatory Roles of Long Non-Coding RNAs in the Central Nervous System and Associated Neurodegenerative Diseases. Front Cell Neurosci 2017;11:175.

19 Bar C, Chatterjee S, Thum T: Long Noncoding RNAs in Cardiovascular Pathology, Diagnosis, and Therapy. Circulation 2016;134:1484-1499. 


\section{Cellular Physiology Cell Physiol Biochem 2018;50:2157-2175 and Biochemistry \begin{tabular}{l|l} 
DOI: 10.1159/000495058 \\
Published online; 10 November 2018
\end{tabular} $\begin{aligned} & \text { @ } 2018 \text { The Author(s). Published by S. Karger AG, Basel } \\
& \text { www.karger.com/cpb }\end{aligned}$

20 Xu N, Chen F, Wang F, Lu X, Wang X, Lv M, Lu C: Clinical significance of high expression of circulating serum lncRNA RP11-445H22.4 in breast cancer patients: a Chinese population-based study. Tumour Biol 2015;36:7659-7665.

-21 Zhang Y, Sun L, Xuan L, Pan Z, Li K, Liu S, Huang Y, Zhao X, Huang L, Wang Z, Hou Y, Li J, Tian Y, Yu J, Han H, Liu Y, Gao F, Zhang Y, Wang S, Du Z, Lu Y, Yang B: Reciprocal Changes of Circulating Long Non-Coding RNAs ZFAS1 and CDR1AS Predict Acute Myocardial Infarction. Sci Rep 2016;6:22384.

-22 Repici M, Centeno C, Tomasi S, Forloni G, Bonny C, Vercelli A, Borsello T: Time-course of c-Jun N-terminal kinase activation after cerebral ischemia and effect of D-JNKI1 on c-Jun and caspase-3 activation. Neuroscience 2007;150:40-49.

-23 Qiu J, Yan Z, Tao K, Li Y, Li Y, Li J, Dong Y, Feng D, Chen H: Sinomenine activates astrocytic dopamine D2 receptors and alleviates neuroinflammatory injury via the CRYAB/STAT3 pathway after ischemic stroke in mice. J Neuroinflammation 2016;13:263.

-24 Valadkhan S, Valencia-Hipolito A: IncRNAs in Stress Response. Curr Top Microbiol Immunol 2016;394:203236.

25 Kim C, Kang D, Lee EK, Lee JS: Long Noncoding RNAs and RNA-Binding Proteins in Oxidative Stress, Cellular Senescence, and Age-Related Diseases. Oxid Med Cell Longev 2017;2017:2062384.

26 Wu X, Li L, Zhang L, Wu J, Zhou Y, Zhou Y, Zhao Y, Zhao J: Inhibition of thioredoxin-1 with siRNA exacerbates apoptosis by activating the ASK1-JNK/p38 pathway in brain of a stroke model rats. Brain Res 2015;1599:20-31.

-27 Kyriakis JM, Avruch J: Mammalian MAPK signal transduction pathways activated by stress and inflammation: a 10-year update. Physiol Rev 2012;92:689-737.

28 Cargnello M, Roux PP: Activation and function of the MAPKs and their substrates, the MAPK-activated protein kinases. Microbiol Mol Biol Rev 2011;75:50-83.

-29 Kong LL, Wang ZY, Han N, Zhuang XM, Wang ZZ, Li H, Chen NH: Neutralization of chemokine-like factor 1, a novel C-C chemokine, protects against focal cerebral ischemia by inhibiting neutrophil infiltration via MAPK pathways in rats. J Neuroinflammation 2014;11:112.

30 Liu X, Ye M, An C, Pan L, Ji L: The effect of cationic albumin-conjugated PEGylated tanshinone IIA nanoparticles on neuronal signal pathways and neuroprotection in cerebral ischemia. Biomaterials 2013;34:6893-6905.

-31 Li Y, Huang J, He X, Tang G, Tang YH, Liu Y, Lin X, Lu Y, Yang GY, Wang Y: Postacute stromal cell-derived factor-1alpha expression promotes neurovascular recovery in ischemic mice. Stroke 2014;45:1822-1829.

-32 Lin Y, Zhang JC, Yao CY, Wu Y, Abdelgawad AF, Yao SL, Yuan SY: Critical role of astrocytic interleukin-17 A in post-stroke survival and neuronal differentiation of neural precursor cells in adult mice. Cell Death Dis 2016; 7:e2273.

33 Jie P, Hong Z, Tian Y, Li Y, Lin L, Zhou L, Du Y, Chen L, Chen L: Activation of transient receptor potential vanilloid 4 induces apoptosis in hippocampus through downregulating PI3K/Akt and upregulating p38 MAPK signaling pathways. Cell Death Dis 2015;6:e1775.

34 Wang P, He Y, Li D, Han R, Liu G, Kong D, Hao J: Class I PI3K inhibitor ZSTK474 mediates a shift in microglial/macrophage phenotype and inhibits inflammatory response in mice with cerebral ischemia/ reperfusion injury. J Neuroinflammation 2016;13:192.

-35 Kilic U, Caglayan AB, Beker MC, Gunal MY, Caglayan B, Yalcin E, Kelestemur T, Gundogdu RZ, Yulug B, Yilmaz B, Kerman BE, Kilic E: Particular phosphorylation of PI3K/Akt on Thr308 via PDK-1 and PTEN mediates melatonin's neuroprotective activity after focal cerebral ischemia in mice. Redox Biol 2017;12:657-665.

-36 Hollander MC, Latour LL, Yang D, Ishii H, Xiao Z, Min Y, Ray-Choudhury A, Munasinghe J, Merchant AS, Lin PC, Hallenbeck J, Boehm M, Yang L: Attenuation of Myeloid-Specific TGFbeta Signaling Induces Inflammatory Cerebrovascular Disease and Stroke. Circ Res 2017;121:1360-1369.

37 Li F, Lan Y, Wang Y, Wang J, Yang G, Meng F, Han H, Meng A, Wang Y, Yang X: Endothelial Smad4 maintains cerebrovascular integrity by activating N-cadherin through cooperation with Notch. Dev Cell 2011;20:291302.

-38 Galluzzi L, Blomgren K, Kroemer G: Mitochondrial membrane permeabilization in neuronal injury. Nat Rev Neurosci 2009;10:481-494.

39 Balaganapathy P, Baik SH, Mallilankaraman K, Sobey CG, Jo DG, Arumugam TV: Interplay between Notch and p53 promotes neuronal cell death in ischemic stroke. J Cereb Blood Flow Metab 2017;10.1177/0271678X17715956271678X17715956. 


\section{Cellular Physiology Cell Physiol Biochem 2018;50:2157-2175 \begin{tabular}{l|l|l} 
and Biochemistry & $\begin{array}{l}\text { DOl: 10.1159/000495058 } \\
\text { Published online: 10 November } 2018\end{array}$ & $\begin{array}{l}\text { O } 2018 \text { The Author(s). Published by S. Karger AG, Basel } \\
\text { www.karger.com/cpb }\end{array}$ \\
\hline
\end{tabular}

40 Li X, Zhao Y, Xia Q, Zheng L, Liu L, Zhao B, Shi J: Nuclear translocation of annexin 1 following oxygen-glucose deprivation-reperfusion induces apoptosis by regulating Bid expression via p53 binding. Cell Death Dis 2016; 7:e2356.

41 Lambert C, Cisternas P, Inestrosa NC: Role of Wnt Signaling in Central Nervous System Injury. Mol Neurobiol 2016;53:2297-2311.

42 Zlokovic BV: Neurovascular pathways to neurodegeneration in Alzheimer's disease and other disorders. Nat Rev Neurosci 2011;12:723-738.

43 Wei ZZ, Zhang JY, Taylor TM, Gu X, Zhao Y, Wei L: Neuroprotective and regenerative roles of intranasal Wnt3a Administration after focal ischemic stroke in mice. J Cereb Blood Flow Metab 2017;10.1177/0271678X1 $7702669271678 X 17702669$.

44 Chang J, Mancuso MR, Maier C, Liang X, Yuki K, Yang L, Kwong JW, Wang J, Rao V, Vallon M, Kosinski C, Zhang JJ, Mah AT, Xu L, Li L, Gholamin S, Reyes TF, Li R, Kuhnert F, Han X et al.: Gpr124 is essential for blood-brain barrier integrity in central nervous system disease. Nat Med 2017;23:450-460.

45 Liu Y, Tang G, Li Y, Wang Y, Chen X, Gu X, Zhang Z, Wang Y, Yang GY: Metformin attenuates blood-brain barrier disruption in mice following middle cerebral artery occlusion. J Neuroinflammation 2014;11:177. 